\title{
Characterization across a dispersity: polymer mass spectrometry in the second dimension
}

\author{
by
}

Tomos E. Morgan ${ }^{1}$, Christopher A. Wootton ${ }^{1}$, Bryan Marzullo ${ }^{1}$, Johanna Paris ${ }^{1}$, Andrew

Kerr $^{1}$, Sean H. Ellacott ${ }^{1}$, Maria A. van Agthoven ${ }^{1,2}$, Mark P. Barrow ${ }^{1}$, Anthony W. T.

Bristow $^{3}$, Sebastien Perrier ${ }^{1,4,5}$, Peter B. O'Connor ${ }^{1 *}$

${ }^{1}$ Department of Chemistry, University of Warwick, Coventry, West Midlands, CV4 7AL, UK.
2Present address: Institute for Organic Chemistry, University of Innsbruck, 80/82 Innrain, 6020,
Innsbruck, Austria
${ }^{3}$ Chemical Development, Pharmaceutical Technology \& Development, Operations, AstraZeneca,
Macclesfield, SK10 2NA, UK.

${ }^{4}$ Warwick Medical School, University of Warwick, Coventry, CV4 7AL, UK

${ }^{5}$ Faculty of Pharmacy and Pharmaceutical Sciences, Monash University, 381 Royal Parade, Parkville, VIC 3052, Australia

*Corresponding authors: Peter O’Connor p.oconnor@warwick.ac.uk

\section{Contents}

Synthesis of Acrylamide species

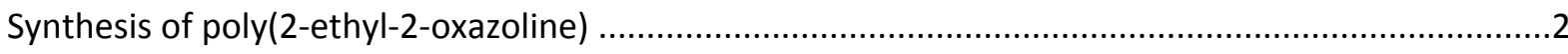

Table S 1: Autocorrelation line assignments of the Poly(2-ethyl-2-oxazoline) species .......................4

Table S 2: Mass spectrometry assignments of the 21-mer polyoxazoline polymer fragment line, Figure $1 \mathrm{C}$ in main text.

Table S 3: Mass spectrometry assignments of the 21-mer polyoxazoline end group losses, Figure 1C in main text.

Table S 4: Mass spectrometry assignments of the 24-mer polyoxazoline polymer fragment line, Figure $1 \mathrm{E}$ in main text.

Table S 5: Mass spectrometry assignments of the 24-mer polyoxazoline end group losses, Figure $1 \mathrm{E}$ in main text.

Table S 6: Mass spectrometry assignments of the H-terminated Polyoxazoline fragments Figure 2 in Main text

Table S 7: Mass spectrometry assignments of the polyacrylamide fragments Figure 5 in Main text..12 


\section{Synthesis of Acrylamide species}

N-N-dimethylacrylamide (DMA, Sigma Aldrich, 99\%), and N-Acryloylmorpoline (NAM, Sigma Aldrich, 97\%) were passed through a column of basic alumina prior to use. 1,4-dioxane (Fisher Scientific), dimethyl sulfoxide- $d_{6}$ (Sigma Aldrich, 99.9\% D atom), chloroform-d (Sigma Aldrich, 99.8\% D atom), and 2,2'-azobis[2-(2-imidazolin-2-yl)propane]dihydrochloride (VA-044, >98\%, Wako) were used as received. 2-(((butylthio)carbonothioyl)thio) propanoic acid (PABTC) was synthesised according to the literature procedure. ${ }^{1}$

\section{Synthesis of poly(2-ethyl-2-oxazoline)}

Synthesis of poly(2-ethyl-2-oxazoline): 2-ethyl-2-oxazoline (99\%, Sigma Aldrich, Dorset, United Kingdom EtOx) was dried over barium oxide and distilled under reduced pressure then kept in a Schlenk flask prior to use. Methyl p-toluenesulfonate (98\%, VWR International Ltd., Lutterworth, United Kingdom), MeTos was distilled under reduced pressure and kept under a nitrogen atmosphere.

Potassium ethyl xanthogenae (96\%, Sigma Aldrich, Dorset, United Kingdom, potassium ethyl xanthate) Acetonitrile Extra Dry $(99.9 \%+$, Fisher Scientific, Acros Organics, Loughborough, United Kingdom) were used as purchased. ${ }^{1} \mathrm{H}$ NMR spectra were measured using Bruker DPX-400 NMR spectrometer which operated at $400.05 \mathrm{MHz}$. Size exclusion chromatography (SEC) measurements in chloroform $\left(\mathrm{CHCl}_{3}\right)$ were performed using an Agilent 390-LC MDS (Agilent Technologies LDA UK, Cheadle, United Kingdom) with differential refractive index (DRI), viscometry (VS), dual-angle light scatter (LS) and two wavelength UV detectors. The system was equipped with $2 \times$ PLgel Mixed D columns $\left(300 \times 7.5 \mathrm{~mm}\right.$, linear operating range between 200 and 400,000 $\left.\mathrm{g} \mathrm{mol}^{-1}\right)$ and a PLgel $5 \mu \mathrm{m}$ guard column (Agilent Technologies LDA UK, Cheadle, United Kingdom). The eluent was $\mathrm{CHCl}_{3}$ with 2\% triethylamine additive. SEC used Polystyrene standards (Agilent Easy Vials (Agilent Technologies LDA UK, Cheadle, United Kingdom) were used for calibration (150-350,000 $\mathrm{g} \mathrm{mol}^{-1}$ ).

Polymerisations of 2-ethyl-2-oxazoline were carried on a Biotage Initiator+ microwave synthesizer (Biotage, Uppsala, Sweden). Dry methyl tosylate (0.186 g, $1 \mathrm{mmol})$, dry 2-ethyl-2-oxazoline (1.983 g, $20 \mathrm{mmol})$ and extra dry acetonitrile $(2.83 \mathrm{~mL})$ were added to a pre-dried Biotage microwave vial, under a constant flux of nitrogen. The vial was sealed, left to stir for $30 \mathrm{~s}$ before being heated at 140 ${ }^{\circ} \mathrm{C}$ for $3 \mathrm{~min}$. After cooling, a $2 \mathrm{~mL}$ solution of potassium ethyl xanthate $(0.192 \mathrm{~g}, 1.2 \mathrm{mmol})$ in extra dry acetonitrile was added with a syringe to the polymer mixture for end-capping of the polymer. The solution was left stirring at room temperature for $48 \mathrm{~h}$. Chloroform $(50 \mathrm{~mL})$ was and the organic phase was washed three times with a saturated solution of sodium hydrogen carbonate, with brine and dried on magnesium sulfate. The polymer was reconstituted in $10 \mathrm{~mL}$ of dichloromethane before precipitation in diethyl ether and dried overnight in a vacuum oven at $40{ }^{\circ} \mathrm{C}$ (yield $1.44 \mathrm{~g}$ ). 
Supporting Information to Characterisation across a dispersity: polymer mass spectrometry in the second dimension

Equations S1-3 shows the calculation of the fractional Kendrick mass calculation (or high resolution Kendrick mass defect (HRMKMr)). The $\mathrm{m} / \mathrm{z}$ values are the monoisotopic mass and charge from SNAP peak picking of the $2 \mathrm{D}$ autocorrelation or fragment lines.

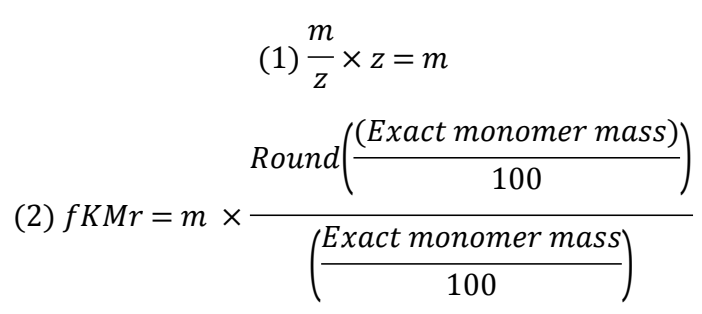

(3) $f K M=f K M r-\operatorname{Round}(f K M r)$

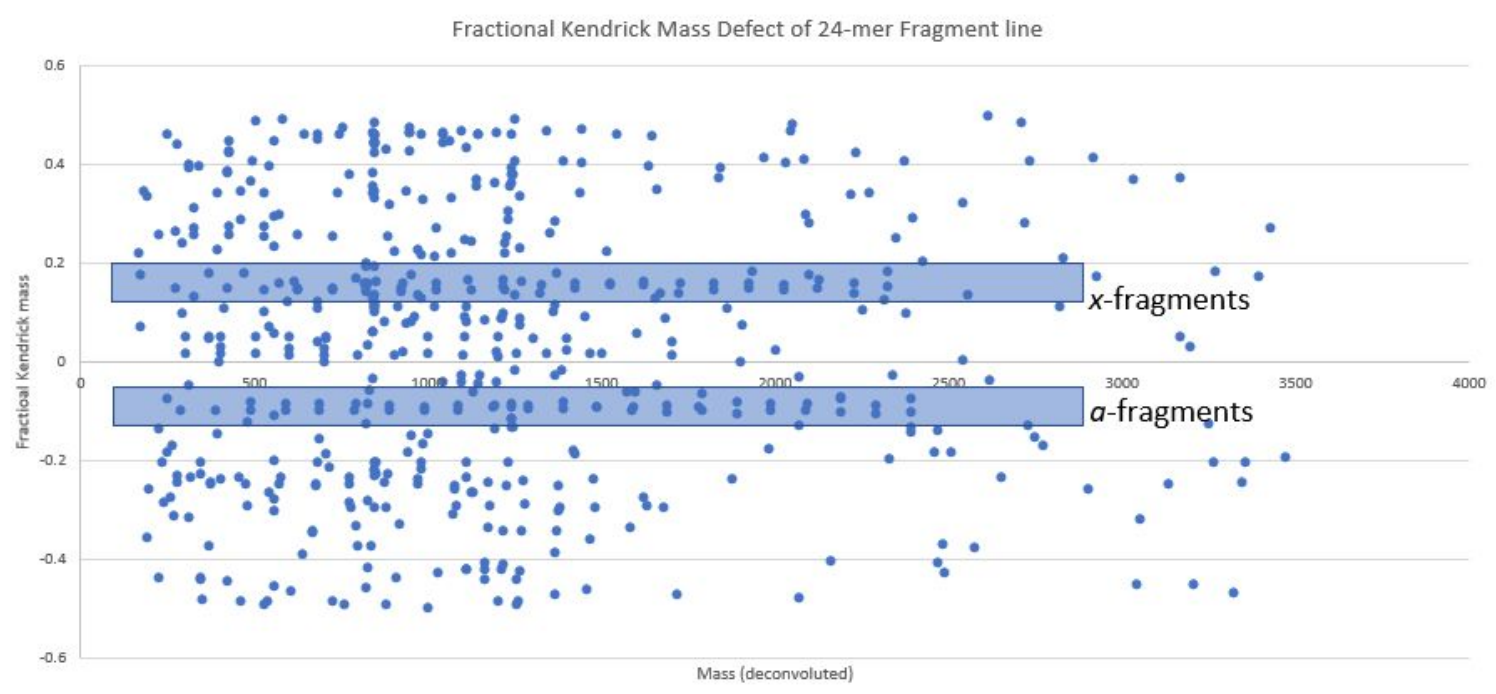

Chart S1: fKM plot of the 24-mer fragment line, with areas containing the $a$ and $x$-fragments highlighted. fKM plotting of fragment lines greatly speeds up mass assignment. 
Supporting Information to Characterisation across a dispersity: polymer mass spectrometry in the second dimension

Table S 1: Autocorrelation line assignments of the Poly(2-ethyl-2-oxazoline) species

\begin{tabular}{|c|c|c|c|c|c|}
\hline $\mathrm{m} / \mathrm{z}$ & z & $\begin{array}{l}\text { Chemical formula } \\
\text { assigned }\end{array}$ & $\begin{array}{l}\text { Monomer } \\
\text { units }\end{array}$ & $\begin{array}{l}\text { error } \\
\text { (ppm) }\end{array}$ & Description \\
\hline 607.72887 & 3 & $\mathrm{C}_{89} \mathrm{H}_{161} \mathrm{~N}_{17} \mathrm{O}_{18} \mathrm{~S}_{2}\left[\mathrm{H}^{+}\right]_{3}$ & 17 & 0.05 & $\mathrm{CH}_{3}$-int, $\mathrm{C}_{3} \mathrm{H}_{5} \mathrm{OS}_{2}$-ter, $3\left[\mathrm{H}^{+}\right]$ \\
\hline 640.75167 & 3 & $\mathrm{C}_{94} \mathrm{H}_{170} \mathrm{~N}_{18} \mathrm{O}_{19} \mathrm{~S}_{2}\left[\mathrm{H}^{+}\right]_{3}$ & 18 & 0.04 & $\mathrm{CH}_{3}$-int, $\mathrm{C}_{3} \mathrm{H}_{5} \mathrm{OS}_{2}$-ter, $3\left[\mathrm{H}^{+}\right]$ \\
\hline 673.77416 & 3 & $\mathrm{C}_{99} \mathrm{H}_{179} \mathrm{~N}_{19} \mathrm{O}_{20} \mathrm{~S}_{2}\left[\mathrm{H}^{+}\right]_{3}$ & 19 & -0.43 & $\mathrm{CH}_{3}$-int, $\mathrm{C}_{3} \mathrm{H}_{5} \mathrm{OS}_{2}$-ter, $3\left[\mathrm{H}^{+}\right]$ \\
\hline 706.79703 & 3 & $\mathrm{C}_{104} \mathrm{H}_{188} \mathrm{~N}_{20} \mathrm{O}_{21} \mathrm{~S}_{2}\left[\mathrm{H}^{+}\right]_{3}$ & 20 & -0.32 & $\mathrm{CH}_{3}$-int, $\mathrm{C}_{3} \mathrm{H}_{5} \mathrm{OS}_{2}$-ter, $3\left[\mathrm{H}^{+}\right]$ \\
\hline 739.81993 & 3 & $\mathrm{C}_{109} \mathrm{H}_{197} \mathrm{~N}_{21} \mathrm{O}_{22} \mathrm{~S}_{2}\left[\mathrm{H}^{+}\right]_{3}$ & 21 & -0.18 & $\mathrm{CH}_{3}$-int, $\mathrm{C}_{3} \mathrm{H}_{5} \mathrm{OS}_{2}$-ter, $3\left[\mathrm{H}^{+}\right]$ \\
\hline 772.84265 & 3 & $\mathrm{C}_{114} \mathrm{H}_{206} \mathrm{~N}_{22} \mathrm{O}_{23} \mathrm{~S}_{2}\left[\mathrm{H}^{+}\right]_{3}$ & 22 & -0.28 & $\mathrm{CH}_{3}$-int, $\mathrm{C}_{3} \mathrm{H}_{5} \mathrm{OS}_{2}$-ter, $3\left[\mathrm{H}^{+}\right]$ \\
\hline 805.86562 & 3 & $\mathrm{C}_{119} \mathrm{H}_{215} \mathrm{~N}_{23} \mathrm{O}_{24} \mathrm{~S}_{2}\left[\mathrm{H}^{+}\right]_{3}$ & 23 & -0.06 & $\mathrm{CH}_{3}$-int, $\mathrm{C}_{3} \mathrm{H}_{5} \mathrm{OS}_{2}$-ter, $3\left[\mathrm{H}^{+}\right]$ \\
\hline 838.88836 & 3 & $\mathrm{C}_{124} \mathrm{H}_{224} \mathrm{~N}_{24} \mathrm{O}_{25} \mathrm{~S}_{2}\left[\mathrm{H}^{+}\right]_{3}$ & 24 & -0.14 & $\mathrm{CH}_{3}$-int, $\mathrm{C}_{3} \mathrm{H}_{5} \mathrm{OS}_{2}$-ter, $3\left[\mathrm{H}^{+}\right]$ \\
\hline 871.91102 & 3 & $\mathrm{C}_{129} \mathrm{H}_{233} \mathrm{~N}_{25} \mathrm{O}_{26} \mathrm{~S}_{2}\left[\mathrm{H}^{+}\right]_{3}$ & 25 & -0.3 & $\mathrm{CH}_{3}$-int, $\mathrm{C}_{3} \mathrm{H}_{5} \mathrm{OS}_{2}$-ter, $3\left[\mathrm{H}^{+}\right]$ \\
\hline 904.93409 & 3 & $\mathrm{C}_{134} \mathrm{H}_{242} \mathrm{~N}_{26} \mathrm{O}_{27} \mathrm{~S}_{2}\left[\mathrm{H}^{+}\right]_{3}$ & 26 & 0.01 & $\mathrm{CH}_{3}$-int, $\mathrm{C}_{3} \mathrm{H}_{5} \mathrm{OS}_{2}$-ter, $3\left[\mathrm{H}^{+}\right]$ \\
\hline 937.95681 & 3 & $\mathrm{C}_{139} \mathrm{H}_{251} \mathrm{~N}_{27} \mathrm{O}_{28} \mathrm{~S}_{2}\left[\mathrm{H}^{+}\right]_{3}$ & 27 & -0.08 & $\mathrm{CH}_{3}$-int, $\mathrm{C}_{3} \mathrm{H}_{5} \mathrm{OS}_{2}$-ter, $3\left[\mathrm{H}^{+}\right]$ \\
\hline 970.97998 & 3 & $\mathrm{C}_{144} \mathrm{H}_{260} \mathrm{~N}_{28} \mathrm{O}_{29} \mathrm{~S}_{2}\left[\mathrm{H}^{+}\right]_{3}$ & 28 & 0.3 & $\mathrm{CH}_{3}$-int, $\mathrm{C}_{3} \mathrm{H}_{5} \mathrm{OS}_{2}$-ter, $3\left[\mathrm{H}^{+}\right]$ \\
\hline 1004.00303 & 3 & $\mathrm{C}_{149} \mathrm{H}_{269} \mathrm{~N}_{29} \mathrm{O}_{30} \mathrm{~S}_{2}\left[\mathrm{H}^{+}\right]_{3}$ & 29 & 0.53 & $\mathrm{CH}_{3}$-int, $\mathrm{C}_{3} \mathrm{H}_{5} \mathrm{OS}_{2}$-ter, $3\left[\mathrm{H}^{+}\right]$ \\
\hline 1037.02629 & 3 & $\mathrm{C}_{154} \mathrm{H}_{278} \mathrm{~N}_{30} \mathrm{O}_{31} \mathrm{~S}_{2}\left[\mathrm{H}^{+}\right]_{3}$ & 30 & 0.95 & $\mathrm{CH}_{3}$-int, $\mathrm{C}_{3} \mathrm{H}_{5} \mathrm{OS}_{2}$-ter, $3\left[\mathrm{H}^{+}\right]$ \\
\hline 1070.04886 & 3 & $\mathrm{C}_{159} \mathrm{H}_{287} \mathrm{~N}_{31} \mathrm{O}_{32} \mathrm{~S}_{2}\left[\mathrm{H}^{+}\right]_{3}$ & 31 & 0.7 & $\mathrm{CH}_{3}$-int, $\mathrm{C}_{3} \mathrm{H}_{5} \mathrm{OS}_{2}$-ter, $3\left[\mathrm{H}^{+}\right]$ \\
\hline 1103.07163 & 3 & $\mathrm{C}_{164} \mathrm{H}_{296} \mathrm{~N}_{32} \mathrm{O}_{33} \mathrm{~S}_{2}\left[\mathrm{H}^{+}\right]_{3}$ & 32 & 0.65 & $\mathrm{CH}_{3}$-int, $\mathrm{C}_{3} \mathrm{H}_{5} \mathrm{OS}_{2}$-ter, $3\left[\mathrm{H}^{+}\right]$ \\
\hline 1136.09381 & 3 & $\mathrm{C}_{169} \mathrm{H}_{305} \mathrm{~N}_{33} \mathrm{O}_{34} \mathrm{~S}_{2}\left[\mathrm{H}^{+}\right]_{3}$ & 33 & 0.08 & $\mathrm{CH}_{3}$-int, $\mathrm{C}_{3} \mathrm{H}_{5} \mathrm{OS}_{2}$-ter, $3\left[\mathrm{H}^{+}\right]$ \\
\hline 712.95295 & 2 & $\mathrm{C}_{69} \mathrm{H}_{125} \mathrm{~N}_{13} \mathrm{O}_{14} \mathrm{~S}_{2}\left[\mathrm{H}^{+}\right]_{2}$ & 13 & 0.22 & $\mathrm{CH}_{3}$-int, $\mathrm{C}_{3} \mathrm{H}_{5} \mathrm{OS}_{2}$-ter, $2\left[\mathrm{H}^{+}\right]$ \\
\hline 762.48684 & 2 & $\mathrm{C}_{74} \mathrm{H}_{134} \mathrm{~N}_{14} \mathrm{O}_{15} \mathrm{~S}_{2}\left[\mathrm{H}^{+}\right]_{2}$ & 14 & -0.21 & $\mathrm{CH}_{3}$-int, $\mathrm{C}_{3} \mathrm{H}_{5} \mathrm{OS}_{2}$-ter, $2\left[\mathrm{H}^{+}\right]$ \\
\hline 812.02075 & 2 & $\mathrm{C}_{79} \mathrm{H}_{143} \mathrm{~N}_{15} \mathrm{O}_{16} \mathrm{~S}_{2}\left[\mathrm{H}^{+}\right]_{2}$ & 15 & -0.57 & $\mathrm{CH}_{3}$-int, $\mathrm{C}_{3} \mathrm{H}_{5} \mathrm{OS}_{2}$-ter, $2\left[\mathrm{H}^{+}\right]$ \\
\hline 861.5552 & 2 & $\mathrm{C}_{84} \mathrm{H}_{152} \mathrm{~N}_{16} \mathrm{O}_{17} \mathrm{~S}_{2}\left[\mathrm{H}^{+}\right]_{2}$ & 16 & -0.25 & $\mathrm{CH}_{3}$-int, $\mathrm{C}_{3} \mathrm{H}_{5} \mathrm{OS}_{2}$-ter, $2\left[\mathrm{H}^{+}\right]$ \\
\hline 911.08938 & 2 & $\mathrm{C}_{89} \mathrm{H}_{161} \mathrm{~N}_{17} \mathrm{O}_{18} \mathrm{~S}_{2}\left[\mathrm{H}^{+}\right]_{2}$ & 17 & -0.27 & $\mathrm{CH}_{3}$-int, $\mathrm{C}_{3} \mathrm{H}_{5} \mathrm{OS}_{2}$-ter, $2\left[\mathrm{H}^{+}\right]$ \\
\hline 960.62383 & 2 & $\mathrm{C}_{94} \mathrm{H}_{170} \mathrm{~N}_{18} \mathrm{O}_{19} \mathrm{~S}_{2}\left[\mathrm{H}^{+}\right]_{2}$ & 18 & 0 & $\mathrm{CH}_{3}$-int, $\mathrm{C}_{3} \mathrm{H}_{5} \mathrm{OS}_{2}$-ter, $2\left[\mathrm{H}^{+}\right]$ \\
\hline 1010.15765 & 2 & $\mathrm{C}_{99} \mathrm{H}_{179} \mathrm{~N}_{19} \mathrm{O}_{20} \mathrm{~S}_{2}\left[\mathrm{H}^{+}\right]_{2}$ & 19 & -0.38 & $\mathrm{CH}_{3}$-int, $\mathrm{C}_{3} \mathrm{H}_{5} \mathrm{OS}_{2}$-ter, $2\left[\mathrm{H}^{+}\right]$ \\
\hline 1059.69282 & 2 & $\mathrm{C}_{104} \mathrm{H}_{188} \mathrm{~N}_{20} \mathrm{O}_{21} \mathrm{~S}_{2}\left[\mathrm{H}^{+}\right]_{2}$ & 20 & 0.54 & $\mathrm{CH}_{3}$-int, $\mathrm{C}_{3} \mathrm{H}_{5} \mathrm{OS}_{2}$-ter, $2\left[\mathrm{H}^{+}\right]$ \\
\hline 1109.22585 & 2 & $\mathrm{C}_{109} \mathrm{H}_{197} \mathrm{~N}_{21} \mathrm{O}_{22} \mathrm{~S}_{2}\left[\mathrm{H}^{+}\right]_{2}$ & 21 & -0.54 & $\mathrm{CH}_{3}$-int, $\mathrm{C}_{3} \mathrm{H}_{5} \mathrm{OS}_{2}$-ter, $2\left[\mathrm{H}^{+}\right]$ \\
\hline 1158.76009 & 2 & $\mathrm{C}_{114} \mathrm{H}_{206} \mathrm{~N}_{22} \mathrm{O}_{23} \mathrm{~S}_{2}\left[\mathrm{H}^{+}\right]_{2}$ & 22 & -0.49 & $\mathrm{CH}_{3}$-int, $\mathrm{C}_{3} \mathrm{H}_{5} \mathrm{OS}_{2}$-ter, $2\left[\mathrm{H}^{+}\right]$ \\
\hline 1208.29407 & 2 & $\mathrm{C}_{119} \mathrm{H}_{215} \mathrm{~N}_{23} \mathrm{O}_{24} \mathrm{~S}_{2}\left[\mathrm{H}^{+}\right]_{2}$ & 23 & -0.66 & $\mathrm{CH}_{3}$-int, $\mathrm{C}_{3} \mathrm{H}_{5} \mathrm{OS}_{2}$-ter, $2\left[\mathrm{H}^{+}\right]$ \\
\hline 1257.82954 & 2 & $\mathrm{C}_{124} \mathrm{H}_{224} \mathrm{~N}_{24} \mathrm{O}_{25} \mathrm{~S}_{2}\left[\mathrm{H}^{+}\right]_{2}$ & 24 & 0.37 & $\mathrm{CH}_{3}$-int, $\mathrm{C}_{3} \mathrm{H}_{5} \mathrm{OS}_{2}$-ter, $2\left[\mathrm{H}^{+}\right]$ \\
\hline 1307.36277 & 2 & $\mathrm{C}_{129} \mathrm{H}_{233} \mathrm{~N}_{25} \mathrm{O}_{26} \mathrm{~S}_{2}\left[\mathrm{H}^{+}\right]_{2}$ & 25 & -0.39 & $\mathrm{CH}_{3}$-int, $\mathrm{C}_{3} \mathrm{H}_{5} \mathrm{OS}_{2}$-ter, $2\left[\mathrm{H}^{+}\right]$ \\
\hline 1356.89608 & 2 & $\mathrm{C}_{134} \mathrm{H}_{242} \mathrm{~N}_{26} \mathrm{O}_{27} \mathrm{~S}_{2}\left[\mathrm{H}^{+}\right]_{2}$ & 26 & -1.04 & $\mathrm{CH}_{3}$-int, $\mathrm{C}_{3} \mathrm{H}_{5} \mathrm{OS}_{2}$-ter, $2\left[\mathrm{H}^{+}\right]$ \\
\hline 1406.43348 & 2 & $\mathrm{C}_{139} \mathrm{H}_{251} \mathrm{~N}_{27} \mathrm{O}_{28} \mathrm{~S}_{2}\left[\mathrm{H}^{+}\right]_{2}$ & 27 & 1.27 & $\mathrm{CH}_{3}$-int, $\mathrm{C}_{3} \mathrm{H}_{5} \mathrm{OS}_{2}$-ter, $2\left[\mathrm{H}^{+}\right]$ \\
\hline 1455.96511 & 2 & $\mathrm{C}_{144} \mathrm{H}_{260} \mathrm{~N}_{28} \mathrm{O}_{29} \mathrm{~S}_{2}\left[\mathrm{H}^{+}\right]_{2}$ & 28 & -0.54 & $\mathrm{CH}_{3}$-int, $\mathrm{C}_{3} \mathrm{H}_{5} \mathrm{OS}_{2}$-ter, $2\left[\mathrm{H}^{+}\right]$ \\
\hline 1505.50268 & 2 & $\mathrm{C}_{149} \mathrm{H}_{269} \mathrm{~N}_{29} \mathrm{O}_{30} \mathrm{~S}_{2}\left[\mathrm{H}^{+}\right]_{2}$ & 29 & 1.71 & $\mathrm{CH}_{3}$-int, $\mathrm{C}_{3} \mathrm{H}_{5} \mathrm{OS}_{2}$-ter, $2\left[\mathrm{H}^{+}\right]$ \\
\hline 1555.03387 & 2 & $\mathrm{C}_{154} \mathrm{H}_{278} \mathrm{~N}_{30} \mathrm{O}_{31} \mathrm{~S}_{2}\left[\mathrm{H}^{+}\right]_{2}$ & 30 & -0.29 & $\mathrm{CH}_{3}$-int, $\mathrm{C}_{3} \mathrm{H}_{5} \mathrm{OS}_{2}$-ter, $2\left[\mathrm{H}^{+}\right]$ \\
\hline 1604.57137 & 2 & $\mathrm{C}_{159} \mathrm{H}_{287} \mathrm{~N}_{31} \mathrm{O}_{32} \mathrm{~S}_{2}\left[\mathrm{H}^{+}\right]_{2}$ & 31 & 1.78 & $\mathrm{CH}_{3}$-int, $\mathrm{C}_{3} \mathrm{H}_{5} \mathrm{OS}_{2}$-ter, $2\left[\mathrm{H}^{+}\right]$ \\
\hline 1212.74621 & 1 & $\mathrm{C}_{58} \mathrm{H}_{105} \mathrm{~N}_{11} \mathrm{O}_{12} \mathrm{~S}_{2}\left[\mathrm{H}^{+}\right]_{1}$ & 11 & 0.31 & $\mathrm{CH}_{3}$-int, $\mathrm{C}_{3} \mathrm{H}_{5} \mathrm{OS}_{2}$-ter, $\left[\mathrm{H}^{+}\right]$ \\
\hline 1410.88215 & 1 & $\mathrm{C}_{68} \mathrm{H}_{123} \mathrm{~N}_{13} \mathrm{O}_{14} \mathrm{~S}_{2}\left[\mathrm{H}^{+}\right]_{1}$ & 13 & -0.36 & $\mathrm{CH}_{3}$-int, $\mathrm{C}_{3} \mathrm{H}_{5} \mathrm{OS}_{2}$-ter, $\left[\mathrm{H}^{+}\right]$ \\
\hline 1509.9504 & 1 & $\mathrm{C}_{73} \mathrm{H}_{132} \mathrm{~N}_{14} \mathrm{O}_{15} \mathrm{~S}_{2}\left[\mathrm{H}^{+}\right]_{1}$ & 14 & -0.45 & $\mathrm{CH}_{3}$-int, $\mathrm{C}_{3} \mathrm{H}_{5} \mathrm{OS}_{2}$-ter, $\left[\mathrm{H}^{+}\right]$ \\
\hline 1609.01955 & 1 & $\mathrm{C}_{78} \mathrm{H}_{141} \mathrm{~N}_{15} \mathrm{O}_{16} \mathrm{~S}_{2}\left[\mathrm{H}^{+}\right]_{1}$ & 15 & 0.04 & $\mathrm{CH}_{3}$-int, $\mathrm{C}_{3} \mathrm{H}_{5} \mathrm{OS}_{2}$-ter, $\left[\mathrm{H}^{+}\right]$ \\
\hline 1708.08835 & 1 & $\mathrm{C}_{83} \mathrm{H}_{150} \mathrm{~N}_{16} \mathrm{O}_{17} \mathrm{~S}_{2}\left[\mathrm{H}^{+}\right]_{1}$ & 16 & 0.26 & $\mathrm{CH}_{3}$-int, $\mathrm{C}_{3} \mathrm{H}_{5} \mathrm{OS}_{2}$-ter, $\left[\mathrm{H}^{+}\right]$ \\
\hline 1807.15703 & 1 & $\mathrm{C}_{88} \mathrm{H}_{159} \mathrm{~N}_{17} \mathrm{O}_{18} \mathrm{~S}_{2}\left[\mathrm{H}^{+}\right]_{1}$ & 17 & 0.39 & $\mathrm{CH}_{3}$-int, $\mathrm{C}_{3} \mathrm{H}_{5} \mathrm{OS}_{2}$-ter, $\left[\mathrm{H}^{+}\right]$ \\
\hline 703.71955 & 4 & $\mathrm{C}_{139} \mathrm{H}_{251} \mathrm{~N}_{27} \mathrm{O}_{28} \mathrm{~S}_{2}\left[\mathrm{H}^{+}\right]_{4}$ & 27 & 0.09 & $\mathrm{CH}_{3}$-int, $\mathrm{C}_{3} \mathrm{H}_{5} \mathrm{OS}_{2}$-ter, $4\left[\mathrm{H}^{+}\right]$ \\
\hline 728.48619 & 4 & $\mathrm{C}_{144} \mathrm{H}_{260} \mathrm{~N}_{28} \mathrm{O}_{29} \mathrm{~S}_{2}\left[\mathrm{H}^{+}\right]_{4}$ & 28 & -0.55 & $\mathrm{CH}_{3}$-int, $\mathrm{C}_{3} \mathrm{H}_{5} \mathrm{OS}_{2}$-ter, $4\left[\mathrm{H}^{+}\right]$ \\
\hline
\end{tabular}


Supporting Information to Characterisation across a dispersity: polymer mass spectrometry in the second dimension

\begin{tabular}{|c|c|c|c|c|}
\hline 753.25351 & 4 & $\mathrm{C}_{149} \mathrm{H}_{269} \mathrm{~N}_{29} \mathrm{O}_{30} \mathrm{~S}_{2}\left[\mathrm{H}^{+}\right]_{4}$ & 29 & -0.24 \\
\hline 778.02029 & 4 & $\mathrm{C}_{154} \mathrm{H}_{278} \mathrm{~N}_{30} \mathrm{O}_{31} \mathrm{~S}_{2}\left[\mathrm{H}^{+}\right]_{4}$ & 30 & -0.65 \\
\hline 802.78815 & 4 & $\mathrm{C}_{159} \mathrm{H}_{287} \mathrm{~N}_{31} \mathrm{O}_{32} \mathrm{~S}_{2}\left[\mathrm{H}^{+}\right]_{4}$ & 31 & 0.31 \\
\hline 827.55504 & 4 & $\mathrm{C}_{164} \mathrm{H}_{296} \mathrm{~N}_{32} \mathrm{O}_{33} \mathrm{~S}_{2}\left[\mathrm{H}^{+}\right]_{4}$ & 32 & 0.05 \\
\hline 852.322 & 4 & $\mathrm{C}_{169} \mathrm{H}_{305} \mathrm{~N}_{33} \mathrm{O}_{34} \mathrm{~S}_{2}\left[\mathrm{H}^{+}\right]_{4}$ & 33 & -0.12 \\
\hline 901.85634 & 4 & $\mathrm{C}_{179} \mathrm{H}_{323} \mathrm{~N}_{35} \mathrm{O}_{36} \mathrm{~S}_{2}\left[\mathrm{H}^{+}\right]_{4}$ & 35 & 0.03 \\
\hline 1135.65895 & 1 & $\mathrm{C}_{53} \mathrm{H}_{96} \mathrm{~N}_{10} \mathrm{O}_{11} \mathrm{~S}_{2}\left[\mathrm{Na}^{+}\right]_{1}$ & 10 & -0.85 \\
\hline 1234.72678 & 1 & $\mathrm{C}_{58} \mathrm{H}_{105} \mathrm{~N}_{11} \mathrm{O}_{12} \mathrm{~S}_{2}\left[\mathrm{Na}^{+}\right]_{1}$ & 11 & -1.25 \\
\hline 1333.79469 & 1 & $\mathrm{C}_{63} \mathrm{H}_{114} \mathrm{~N}_{12} \mathrm{O}_{13} \mathrm{~S}_{2}\left[\mathrm{Na}^{+}\right]_{1}$ & 12 & -1.54 \\
\hline 1432.86364 & 1 & $\mathrm{C}_{68} \mathrm{H}_{123} \mathrm{~N}_{13} \mathrm{O}_{14} \mathrm{~S}_{2}\left[\mathrm{Na}^{+}\right]_{1}$ & 13 & -1.06 \\
\hline 1531.93252 & 1 & $\mathrm{C}_{73} \mathrm{H}_{132} \mathrm{~N}_{14} \mathrm{O}_{15} \mathrm{~S}_{2}\left[\mathrm{Na}^{+}\right]_{1}$ & 14 & -0.69 \\
\hline 1631.00217 & 1 & $\mathrm{C}_{78} \mathrm{H}_{141} \mathrm{~N}_{15} \mathrm{O}_{16} \mathrm{~S}_{2}\left[\mathrm{Na}^{+}\right]_{1}$ & 15 & 0.11 \\
\hline 1730.06942 & 1 & $\mathrm{C}_{83} \mathrm{H}_{150} \mathrm{~N}_{16} \mathrm{O}_{17} \mathrm{~S}_{2}\left[\mathrm{Na}^{+}\right]_{1}$ & 16 & -0.57 \\
\hline 1829.13922 & 1 & $\mathrm{C}_{88} \mathrm{H}_{159} \mathrm{~N}_{17} \mathrm{O}_{18} \mathrm{~S}_{2}\left[\mathrm{Na}^{+}\right]_{1}$ & 17 & 0.22 \\
\hline 1928.20409 & 1 & $\mathrm{C}_{93} \mathrm{H}_{168} \mathrm{~N}_{18} \mathrm{O}_{19} \mathrm{~S}_{2}\left[\mathrm{Na}^{+}\right]_{1}$ & 18 & -1.63 \\
\hline 681.10151 & 3 & $\mathrm{C}_{99} \mathrm{H}_{179} \mathrm{~N}_{19} \mathrm{O}_{20} \mathrm{~S}_{2}\left[\mathrm{H}^{+}\right]_{2}\left[\mathrm{Na}^{+}\right]_{1}$ & 19 & -0.64 \\
\hline 714.12432 & 3 & $\mathrm{C}_{104} \mathrm{H}_{188} \mathrm{~N}_{20} \mathrm{O}_{21} \mathrm{~S}_{2}\left[\mathrm{H}^{+}\right]_{2}\left[\mathrm{Na}^{+}\right]_{1}$ & 20 & -0.61 \\
\hline 747.14725 & 3 & $\mathrm{C}_{109} \mathrm{H}_{197} \mathrm{~N}_{21} \mathrm{O}_{22} \mathrm{~S}_{2}\left[\mathrm{H}^{+}\right]_{2}\left[\mathrm{Na}^{+}\right]_{1}$ & 21 & -0.41 \\
\hline 780.17037 & 3 & $\mathrm{C}_{114} \mathrm{H}_{206} \mathrm{~N}_{22} \mathrm{O}_{23} \mathrm{~S}_{2}\left[\mathrm{H}^{+}\right]_{2}\left[\mathrm{Na}^{+}\right]_{1}$ & 22 & 0.01 \\
\hline 813.19231 & 3 & $\mathrm{C}_{119} \mathrm{H}_{215} \mathrm{~N}_{23} \mathrm{O}_{24} \mathrm{~S}_{2}\left[\mathrm{H}^{+}\right]_{2}\left[\mathrm{Na}^{+}\right]_{1}$ & 23 & -1.05 \\
\hline 846.21541 & 3 & $\mathrm{C}_{124} \mathrm{H}_{224} \mathrm{~N}_{24} \mathrm{O}_{25} \mathrm{~S}_{2}\left[\mathrm{H}^{+}\right]_{2}\left[\mathrm{Na}^{+}\right]_{1}$ & 24 & -0.66 \\
\hline 879.23851 & 3 & $\mathrm{C}_{129} \mathrm{H}_{233} \mathrm{~N}_{25} \mathrm{O}_{26} \mathrm{~S}_{2}\left[\mathrm{H}^{+}\right]_{2}\left[\mathrm{Na}^{+}\right]_{1}$ & 25 & -0.3 \\
\hline 912.26223 & 3 & $\mathrm{C}_{134} \mathrm{H}_{242} \mathrm{~N}_{26} \mathrm{O}_{27} \mathrm{~S}_{2}\left[\mathrm{H}^{+}\right]_{2}\left[\mathrm{Na}^{+}\right]_{1}$ & 26 & 0.71 \\
\hline 945.28422 & 3 & $\mathrm{C}_{139} \mathrm{H}_{251} \mathrm{~N}_{27} \mathrm{O}_{28} \mathrm{~S}_{2}\left[\mathrm{H}^{+}\right]_{2}\left[\mathrm{Na}^{+}\right]_{1}$ & 27 & -0.17 \\
\hline 978.30661 & 3 & $\mathrm{C}_{144} \mathrm{H}_{260} \mathrm{~N}_{28} \mathrm{O}_{29} \mathrm{~S}_{2}\left[\mathrm{H}^{+}\right]_{2}\left[\mathrm{Na}^{+}\right]_{1}$ & 28 & -0.59 \\
\hline 1044.35202 & 3 & $\mathrm{C}_{154} \mathrm{H}_{278} \mathrm{~N}_{30} \mathrm{O}_{31} \mathrm{~S}_{2}\left[\mathrm{H}^{+}\right]_{2}\left[\mathrm{Na}^{+}\right]_{1}$ & 30 & -0.75 \\
\hline 872.54726 & 2 & $\mathrm{C}_{84} \mathrm{H}_{152} \mathrm{~N}_{16} \mathrm{O}_{17} \mathrm{~S}_{2}\left[\mathrm{H}^{+}\right]_{1}\left[\mathrm{Na}^{+}\right]_{1}$ & 16 & 0.68 \\
\hline 922.08033 & 2 & $\mathrm{C}_{89} \mathrm{H}_{161} \mathrm{~N}_{17} \mathrm{O}_{18} \mathrm{~S}_{2}\left[\mathrm{H}^{+}\right]_{1}\left[\mathrm{Na}^{+}\right]_{1}$ & 17 & -0.59 \\
\hline 971.61481 & 2 & $\mathrm{C}_{94} \mathrm{H}_{170} \mathrm{~N}_{18} \mathrm{O}_{19} \mathrm{~S}_{2}\left[\mathrm{H}^{+}\right]_{1}\left[\mathrm{Na}^{+}\right]_{1}$ & 18 & -0.27 \\
\hline 1021.14836 & 2 & $\mathrm{C}_{99} \mathrm{H}_{179} \mathrm{~N}_{19} \mathrm{O}_{20} \mathrm{~S}_{2}\left[\mathrm{H}^{+}\right]_{1}\left[\mathrm{Na}^{+}\right]_{1}$ & 19 & -0.9 \\
\hline 1070.6825 & 2 & $\mathrm{C}_{104} \mathrm{H}_{188} \mathrm{~N}_{20} \mathrm{O}_{21} \mathrm{~S}_{2}\left[\mathrm{H}^{+}\right]_{1}\left[\mathrm{Na}^{+}\right]_{1}$ & 20 & -0.93 \\
\hline 1120.21737 & 2 & $\mathrm{C}_{109} \mathrm{H}_{197} \mathrm{~N}_{21} \mathrm{O}_{22} \mathrm{~S}_{2}\left[\mathrm{H}^{+}\right]_{1}\left[\mathrm{Na}^{+}\right]_{1}$ & 21 & -0.29 \\
\hline 1169.75196 & 2 & $\mathrm{C}_{114} \mathrm{H}_{206} \mathrm{~N}_{22} \mathrm{O}_{23} \mathrm{~S}_{2}\left[\mathrm{H}^{+}\right]_{1}\left[\mathrm{Na}^{+}\right]_{1}$ & 22 & 0.05 \\
\hline 1219.28556 & 2 & $\mathrm{C}_{119} \mathrm{H}_{215} \mathrm{~N}_{23} \mathrm{O}_{24} \mathrm{~S}_{2}\left[\mathrm{H}^{+}\right]_{1}\left[\mathrm{Na}^{+}\right]_{1}$ & 23 & -0.45 \\
\hline 1268.82078 & 2 & $\mathrm{C}_{124} \mathrm{H}_{224} \mathrm{~N}_{24} \mathrm{O}_{25} \mathrm{~S}_{2}\left[\mathrm{H}^{+}\right]_{1}\left[\mathrm{Na}^{+}\right]_{1}$ & 24 & 0.36 \\
\hline 1318.35405 & 2 & $\mathrm{C}_{129} \mathrm{H}_{233} \mathrm{~N}_{25} \mathrm{O}_{26} \mathrm{~S}_{2}\left[\mathrm{H}^{+}\right]_{1}\left[\mathrm{Na}^{+}\right]_{1}$ & 25 & -0.36 \\
\hline 1367.8877 & 2 & $\mathrm{C}_{134} \mathrm{H}_{242} \mathrm{~N}_{26} \mathrm{O}_{27} \mathrm{~S}_{2}\left[\mathrm{H}^{+}\right]_{1}\left[\mathrm{Na}^{+}\right]_{1}$ & 26 & -0.75 \\
\hline 1417.42406 & 2 & $\mathrm{C}_{139} \mathrm{H}_{251} \mathrm{~N}_{27} \mathrm{O}_{28} \mathrm{~S}_{2}\left[\mathrm{H}^{+}\right]_{1}\left[\mathrm{Na}^{+}\right]_{1}$ & 27 & 0.79 \\
\hline 1466.95777 & 2 & $\mathrm{C}_{144} \mathrm{H}_{260} \mathrm{~N}_{28} \mathrm{O}_{29} \mathrm{~S}_{2}\left[\mathrm{H}^{+}\right]_{1}\left[\mathrm{Na}^{+}\right]_{1}$ & 28 & 0.43 \\
\hline 1516.49208 & 2 & $\mathrm{C}_{149} \mathrm{H}_{269} \mathrm{~N}_{29} \mathrm{O}_{30} \mathrm{~S}_{2}\left[\mathrm{H}^{+}\right]_{1}\left[\mathrm{Na}^{+}\right]_{1}$ & 29 & 0.48 \\
\hline 669.10243 & 3 & $\mathrm{C}_{98} \mathrm{H}_{177} \mathrm{~N}_{19} \mathrm{O}_{20} \mathrm{~S}_{2}\left[\mathrm{H}^{+}\right]_{3}$ & 19 & -0.2 \\
\hline 702.12529 & 3 & $\mathrm{C}_{103} \mathrm{H}_{186} \mathrm{~N}_{20} \mathrm{O}_{21} \mathrm{~S}_{2}\left[\mathrm{H}^{+}\right]_{3}$ & 20 & -0.12 \\
\hline 735.14801 & 3 & $\mathrm{C}_{108} \mathrm{H}_{195} \mathrm{~N}_{21} \mathrm{O}_{22} \mathrm{~S}_{2}\left[\mathrm{H}^{+}\right]_{3}$ & 21 & -0.23 \\
\hline 768.17075 & 3 & $\mathrm{C}_{113} \mathrm{H}_{204} \mathrm{~N}_{22} \mathrm{O}_{23} \mathrm{~S}_{2}\left[\mathrm{H}^{+}\right]_{3}$ & 22 & -0.3 \\
\hline 801.19351 & 3 & $\mathrm{C}_{118} \mathrm{H}_{213} \mathrm{~N}_{23} \mathrm{O}_{24} \mathrm{~S}_{2}\left[\mathrm{H}^{+}\right]_{3}$ & 23 & -0.34 \\
\hline 834.21652 & 3 & $\mathrm{C}_{123} \mathrm{H}_{222} \mathrm{~N}_{24} \mathrm{O}_{25} \mathrm{~S}_{2}\left[\mathrm{H}^{+}\right]_{3}$ & 24 & -0.08 \\
\hline 867.2389 & 3 & $\mathrm{C}_{128} \mathrm{H}_{231} \mathrm{~N}_{25} \mathrm{O}_{26} \mathrm{~S}_{2}\left[\mathrm{H}^{+}\right]_{3}$ & 25 & -0.57 \\
\hline
\end{tabular}

$\mathrm{CH}_{3}$-int, $\mathrm{C}_{3} \mathrm{H}_{5} \mathrm{OS}_{2}$-ter, $4\left[\mathrm{H}^{+}\right]$ $\mathrm{CH}_{3}$-int, $\mathrm{C}_{3} \mathrm{H}_{5} \mathrm{OS}_{2}$-ter, $4\left[\mathrm{H}^{+}\right]$ $\mathrm{CH}_{3}$-int, $\mathrm{C}_{3} \mathrm{H}_{5} \mathrm{OS}_{2}$-ter, $4\left[\mathrm{H}^{+}\right]$ $\mathrm{CH}_{3}$-int, $\mathrm{C}_{3} \mathrm{H}_{5} \mathrm{OS}_{2}$-ter, $4\left[\mathrm{H}^{+}\right]$ $\mathrm{CH}_{3}$-int, $\mathrm{C}_{3} \mathrm{H}_{5} \mathrm{OS}_{2}$-ter, $4\left[\mathrm{H}^{+}\right]$ $\mathrm{CH}_{3}$-int, $\mathrm{C}_{3} \mathrm{H}_{5} \mathrm{OS}_{2}$-ter, $4\left[\mathrm{H}^{+}\right]$ $\mathrm{CH}_{3}$-int, $\mathrm{C}_{3} \mathrm{H}_{5} \mathrm{OS}_{2}$-ter, $2\left[\mathrm{H}^{+}\right]\left[\mathrm{Na}^{+}\right]$ $\mathrm{CH}_{3}$-int, $\mathrm{C}_{3} \mathrm{H}_{5} \mathrm{OS}_{2}$-ter, $2\left[\mathrm{H}^{+}\right]\left[\mathrm{Na}^{+}\right]$ $\mathrm{CH}_{3}$-int, $\mathrm{C}_{3} \mathrm{H}_{5} \mathrm{OS}_{2}$-ter, $2\left[\mathrm{H}^{+}\right]\left[\mathrm{Na}^{+}\right]$ $\mathrm{CH}_{3}$-int, $\mathrm{C}_{3} \mathrm{H}_{5} \mathrm{OS}_{2}$-ter, $2\left[\mathrm{H}^{+}\right]\left[\mathrm{Na}^{+}\right]$ $\mathrm{CH}_{3}$-int, $\mathrm{C}_{3} \mathrm{H}_{5} \mathrm{OS}_{2}$-ter, $2\left[\mathrm{H}^{+}\right]\left[\mathrm{Na}^{+}\right]$ $\mathrm{CH}_{3}$-int, $\mathrm{C}_{3} \mathrm{H}_{5} \mathrm{OS}_{2}$-ter, $2\left[\mathrm{H}^{+}\right]\left[\mathrm{Na}^{+}\right]$ $\mathrm{CH}_{3}$-int, $\mathrm{C}_{3} \mathrm{H}_{5} \mathrm{OS}_{2}$-ter, $2\left[\mathrm{H}^{+}\right]\left[\mathrm{Na}^{+}\right]$ $\mathrm{CH}_{3}$-int, $\mathrm{C}_{3} \mathrm{H}_{5} \mathrm{OS}_{2}$-ter, $2\left[\mathrm{H}^{+}\right]\left[\mathrm{Na}^{+}\right]$ $\mathrm{CH}_{3}$-int, $\mathrm{C}_{3} \mathrm{H}_{5} \mathrm{OS}_{2}$-ter, $2\left[\mathrm{H}^{+}\right]\left[\mathrm{Na}^{+}\right]$ $\mathrm{CH}_{3}$-int, $\mathrm{C}_{3} \mathrm{H}_{5} \mathrm{OS}_{2}$-ter, $2\left[\mathrm{H}^{+}\right]\left[\mathrm{Na}^{+}\right]$ $\mathrm{CH}_{3}$-int, $\mathrm{C}_{3} \mathrm{H}_{5} \mathrm{OS}_{2}$-ter, $2\left[\mathrm{H}^{+}\right]\left[\mathrm{Na}^{+}\right]$ $\mathrm{CH}_{3}$-int, $\mathrm{C}_{3} \mathrm{H}_{5} \mathrm{OS}_{2}$-ter, $2\left[\mathrm{H}^{+}\right]\left[\mathrm{Na}^{+}\right]$ $\mathrm{CH}_{3}$-int, $\mathrm{C}_{3} \mathrm{H}_{5} \mathrm{OS}_{2}$-ter, $2\left[\mathrm{H}^{+}\right]\left[\mathrm{Na}^{+}\right]$ $\mathrm{CH}_{3}$-int, $\mathrm{C}_{3} \mathrm{H}_{5} \mathrm{OS}_{2}$-ter, $2\left[\mathrm{H}^{+}\right]\left[\mathrm{Na}^{+}\right]$ $\mathrm{CH}_{3}$-int, $\mathrm{C}_{3} \mathrm{H}_{5} \mathrm{OS}_{2}$-ter, $2\left[\mathrm{H}^{+}\right]\left[\mathrm{Na}^{+}\right]$ $\mathrm{CH}_{3}$-int, $\mathrm{C}_{3} \mathrm{H}_{5} \mathrm{OS}_{2}$-ter, $2\left[\mathrm{H}^{+}\right]\left[\mathrm{Na}^{+}\right]$ $\mathrm{CH}_{3}$-int, $\mathrm{C}_{3} \mathrm{H}_{5} \mathrm{OS}_{2}$-ter, $2\left[\mathrm{H}^{+}\right]\left[\mathrm{Na}^{+}\right]$ $\mathrm{CH}_{3}$-int, $\mathrm{C}_{3} \mathrm{H}_{5} \mathrm{OS}_{2}$-ter, $2\left[\mathrm{H}^{+}\right]\left[\mathrm{Na}^{+}\right]$ $\mathrm{CH}_{3}$-int, $\mathrm{C}_{3} \mathrm{H}_{5} \mathrm{OS}_{2}$-ter, $2\left[\mathrm{H}^{+}\right]\left[\mathrm{Na}^{+}\right]$ $\mathrm{CH}_{3}$-int, $\mathrm{C}_{3} \mathrm{H}_{5} \mathrm{OS}_{2}$-ter, $2\left[\mathrm{H}^{+}\right]\left[\mathrm{Na}^{+}\right]$ $\mathrm{CH}_{3}$-int, $\mathrm{C}_{3} \mathrm{H}_{5} \mathrm{OS}_{2}$-ter, $\left[\mathrm{H}^{+}\right]\left[\mathrm{Na}^{+}\right]$ $\mathrm{CH}_{3}$-int, $\mathrm{C}_{3} \mathrm{H}_{5} \mathrm{OS}_{2}$-ter, $\left[\mathrm{H}^{+}\right]\left[\mathrm{Na}^{+}\right]$ $\mathrm{CH}_{3}$-int, $\mathrm{C}_{3} \mathrm{H}_{5} \mathrm{OS}_{2}$-ter, $\left[\mathrm{H}^{+}\right]\left[\mathrm{Na}^{+}\right]$ $\mathrm{CH}_{3}$-int, $\mathrm{C}_{3} \mathrm{H}_{5} \mathrm{OS}_{2}$-ter, $\left[\mathrm{H}^{+}\right]\left[\mathrm{Na}^{+}\right]$ $\mathrm{CH}_{3}$-int, $\mathrm{C}_{3} \mathrm{H}_{5} \mathrm{OS}_{2}$-ter, $\left[\mathrm{H}^{+}\right]\left[\mathrm{Na}^{+}\right]$ $\mathrm{CH}_{3}$-int, $\mathrm{C}_{3} \mathrm{H}_{5} \mathrm{OS}_{2}$-ter, $\left[\mathrm{H}^{+}\right]\left[\mathrm{Na}^{+}\right]$ $\mathrm{CH}_{3}$-int, $\mathrm{C}_{3} \mathrm{H}_{5} \mathrm{OS}_{2}$-ter, $\left[\mathrm{H}^{+}\right]\left[\mathrm{Na}^{+}\right]$ $\mathrm{CH}_{3}$-int, $\mathrm{C}_{3} \mathrm{H}_{5} \mathrm{OS}_{2}$-ter, $\left[\mathrm{H}^{+}\right]\left[\mathrm{Na}^{+}\right]$ $\mathrm{CH}_{3}$-int, $\mathrm{C}_{3} \mathrm{H}_{5} \mathrm{OS}_{2}$-ter, $\left[\mathrm{H}^{+}\right]\left[\mathrm{Na}^{+}\right]$ $\mathrm{CH}_{3}$-int, $\mathrm{C}_{3} \mathrm{H}_{5} \mathrm{OS}_{2}$-ter, $\left[\mathrm{H}^{+}\right]\left[\mathrm{Na}^{+}\right]$ $\mathrm{CH}_{3}$-int, $\mathrm{C}_{3} \mathrm{H}_{5} \mathrm{OS}_{2}$-ter, $\left[\mathrm{H}^{+}\right]\left[\mathrm{Na}^{+}\right]$ $\mathrm{CH}_{3}$-int, $\mathrm{C}_{3} \mathrm{H}_{5} \mathrm{OS}_{2}$-ter, $\left[\mathrm{H}^{+}\right]\left[\mathrm{Na}^{+}\right]$ $\mathrm{CH}_{3}$-int, $\mathrm{C}_{3} \mathrm{H}_{5} \mathrm{OS}_{2}$-ter, $\left[\mathrm{H}^{+}\right]\left[\mathrm{Na}^{+}\right]$ $\mathrm{CH}_{3}$-int, $\mathrm{C}_{3} \mathrm{H}_{5} \mathrm{OS}_{2}$-ter, $\left[\mathrm{H}^{+}\right]\left[\mathrm{Na}^{+}\right]$ $\mathrm{H}$-int, $\mathrm{C}_{3} \mathrm{H}_{5} \mathrm{OS}_{2}$-ter, $3\left[\mathrm{H}^{+}\right]$ $\mathrm{H}$-int, $\mathrm{C}_{3} \mathrm{H}_{5} \mathrm{OS}_{2}$-ter, $3\left[\mathrm{H}^{+}\right]$ $\mathrm{H}$-int, $\mathrm{C}_{3} \mathrm{H}_{5} \mathrm{OS}_{2}$-ter, $3\left[\mathrm{H}^{+}\right]$ $\mathrm{H}$-int, $\mathrm{C}_{3} \mathrm{H}_{5} \mathrm{OS}_{2}$-ter, $3\left[\mathrm{H}^{+}\right]$ $\mathrm{H}$-int, $\mathrm{C}_{3} \mathrm{H}_{5} \mathrm{OS}_{2}$-ter, $3\left[\mathrm{H}^{+}\right]$ $\mathrm{H}$-int, $\mathrm{C}_{3} \mathrm{H}_{5} \mathrm{OS}_{2}$-ter, $3\left[\mathrm{H}^{+}\right]$ $\mathrm{H}$-int, $\mathrm{C}_{3} \mathrm{H}_{5} \mathrm{OS}_{2}$-ter, $3\left[\mathrm{H}^{+}\right]$ 
Supporting Information to Characterisation across a dispersity: polymer mass spectrometry in the second dimension

$\begin{array}{rllrrr}900.26172 & 3 & \mathrm{C}_{133} \mathrm{H}_{240} \mathrm{~N}_{26} \mathrm{O}_{27} \mathrm{~S}_{2}\left[\mathrm{H}^{+}\right]_{3} & 26 & -0.53 & \mathrm{H} \text {-int, } \mathrm{C}_{3} \mathrm{H}_{5} \mathrm{OS}_{2} \text {-ter, } 3\left[\mathrm{H}^{+}\right] \\ 933.28506 & 3 & \mathrm{C}_{138} \mathrm{H}_{249} \mathrm{~N}_{27} \mathrm{O}_{28} \mathrm{~S}_{2}\left[\mathrm{H}^{+}\right]_{3} & 27 & 0.06 & \mathrm{H} \text {-int, } \mathrm{C}_{3} \mathrm{H}_{5} \mathrm{OS}_{2} \text {-ter, } 3\left[\mathrm{H}^{+}\right] \\ 966.3067 & 3 & \mathrm{C}_{143} \mathrm{H}_{258} \mathrm{~N}_{28} \mathrm{O}_{29} \mathrm{~S}_{2}\left[\mathrm{H}^{+}\right]_{3} & 28 & -1.15 & \mathrm{H} \text {-int, } \mathrm{C}_{3} \mathrm{H}_{5} \mathrm{OS}_{2} \text {-ter, } 3\left[\mathrm{H}^{+}\right] \\ 999.33061 & 3 & \mathrm{C}_{148} \mathrm{H}_{267} \mathrm{~N}_{29} \mathrm{O}_{30} \mathrm{~S}_{2}\left[\mathrm{H}^{+}\right]_{3} & 29 & 0 & \mathrm{H} \text {-int, } \mathrm{C}_{3} \mathrm{H}_{5} \mathrm{OS}_{2} \text {-ter, } 3\left[\mathrm{H}^{+}\right] \\ 1032.35315 & 3 & \mathrm{C}_{153} \mathrm{H}_{276} \mathrm{~N}_{30} \mathrm{O}_{31} \mathrm{~S}_{2}\left[\mathrm{H}^{+}\right]_{3} & 30 & -0.26 & \mathrm{H} \text {-int, } \mathrm{C}_{3} \mathrm{H}_{5} \mathrm{OS}_{2} \text {-ter, } 3\left[\mathrm{H}^{+}\right] \\ & \text {Absolute average } & & 0.47 & \\ & & \text { Std Deviation } & & 0.59 & \end{array}$


Supporting Information to Characterisation across a dispersity: polymer mass spectrometry in the second dimension

Table S 2: Mass spectrometry assignments of the 21-mer polyoxazoline polymer fragment line, Figure $1 \mathrm{C}$ in main text

\begin{tabular}{|c|c|c|c|c|}
\hline $\mathrm{m} / \mathrm{z}$ & charge & chemical formula & $\begin{array}{l}\text { error } \\
\text { (ppm) }\end{array}$ & assignment \\
\hline 187.1443 & 1 & $\mathrm{C}_{9} \mathrm{H}_{18} \mathrm{~N}_{2} \mathrm{O}_{2}\left[\mathrm{H}^{+}\right]_{1}$ & 1.21 & $a_{2}$ \\
\hline 286.2125 & 1 & $\mathrm{C}_{14} \mathrm{H}_{27} \mathrm{~N}_{3} \mathrm{O}_{3}\left[\mathrm{H}^{+}\right]_{1}$ & 0.01 & $a_{3}$ \\
\hline 385.2808 & 1 & $\mathrm{C}_{19} \mathrm{H}_{36} \mathrm{~N}_{4} \mathrm{O}_{4}\left[\mathrm{H}^{+}\right]_{1}$ & -0.39 & $a_{4}$ \\
\hline 484.3493 & 1 & $\mathrm{C}_{24} \mathrm{H}_{45} \mathrm{~N}_{5} \mathrm{O}_{5}\left[\mathrm{H}^{+}\right]_{1}$ & -0.10 & $a_{5}$ \\
\hline 583.4178 & 1 & $\mathrm{C}_{29} \mathrm{H}_{54} \mathrm{~N}_{6} \mathrm{O}_{6}\left[\mathrm{H}^{+}\right]_{1}$ & 0.00 & $a_{6}$ \\
\hline 682.4864 & 1 & $\mathrm{C}_{34} \mathrm{H}_{63} \mathrm{~N}_{7} \mathrm{O}_{7}\left[\mathrm{H}^{+}\right]_{1}$ & 0.27 & $a_{7}$ \\
\hline 781.5547 & 1 & $\mathrm{C}_{39} \mathrm{H}_{72} \mathrm{~N}_{8} \mathrm{O}_{8}\left[\mathrm{H}^{+}\right]_{1}$ & 0.13 & $a_{8}$ \\
\hline 880.6224 & 1 & $\mathrm{C}_{44} \mathrm{H}_{81} \mathrm{~N}_{9} \mathrm{O}_{9}\left[\mathrm{H}^{+}\right]_{1}$ & -0.73 & $a_{9}$ \\
\hline 979.6914 & 1 & $\mathrm{C}_{49} \mathrm{H}_{90} \mathrm{~N}_{10} \mathrm{O}_{10}\left[\mathrm{H}^{+}\right]_{1}$ & -0.07 & $a_{10}$ \\
\hline 1078.761 & 1 & $\mathrm{C}_{54} \mathrm{H}_{99} \mathrm{~N}_{11} \mathrm{O}_{11}\left[\mathrm{H}^{+}\right]_{1}$ & 0.70 & $a_{11}$ \\
\hline 1177.828 & 1 & $\mathrm{C}_{59} \mathrm{H}_{108} \mathrm{~N}_{12} \mathrm{O}_{12}\left[\mathrm{H}^{+}\right]_{1}$ & -0.02 & $a_{12}$ \\
\hline 1276.896 & 1 & $\mathrm{C}_{64} \mathrm{H}_{117} \mathrm{~N}_{13} \mathrm{O}_{13}\left[\mathrm{H}^{+}\right]_{1}$ & -0.13 & $a_{13}$ \\
\hline 1375.965 & 1 & $\mathrm{C}_{69} \mathrm{H}_{126} \mathrm{~N}_{14} \mathrm{O}_{14}\left[\mathrm{H}^{+}\right]_{1}$ & -0.27 & $a_{14}$ \\
\hline 1475.032 & 1 & $\mathrm{C}_{74} \mathrm{H}_{135} \mathrm{~N}_{15} \mathrm{O}_{15}\left[\mathrm{H}^{+}\right]_{1}$ & -0.76 & $a_{15}$ \\
\hline 1574.102 & 1 & $\mathrm{C}_{79} \mathrm{H}_{144} \mathrm{~N}_{16} \mathrm{O}_{16}\left[\mathrm{H}^{+}\right]_{1}$ & -0.09 & $a_{16}$ \\
\hline 1673.171 & 1 & $\mathrm{C}_{84} \mathrm{H}_{153} \mathrm{~N}_{17} \mathrm{O}_{17}\left[\mathrm{H}^{+}\right]_{1}$ & 0.40 & $a_{17}$ \\
\hline 1772.239 & 1 & $\mathrm{C}_{89} \mathrm{H}_{162} \mathrm{~N}_{18} \mathrm{O}_{18}\left[\mathrm{H}^{+}\right]_{1}$ & 0.01 & $a_{18}$ \\
\hline 1871.306 & 1 & $\mathrm{C}_{94} \mathrm{H}_{171} \mathrm{~N}_{19} \mathrm{O}_{19}\left[\mathrm{H}^{+}\right]_{1}$ & -0.56 & $a_{19}$ \\
\hline 1970.376 & 1 & $\mathrm{C}_{99} \mathrm{H}_{180} \mathrm{~N}_{20} \mathrm{O}_{20}\left[\mathrm{H}^{+}\right]_{1}$ & 0.02 & $a_{20}$ \\
\hline 321.1301 & 1 & $\mathrm{C}_{13} \mathrm{H}_{24} \mathrm{~N}_{2} \mathrm{O}_{3} \mathrm{~S}_{2}\left[\mathrm{H}^{+}\right]_{1}$ & 0.03 & $x_{2}$ \\
\hline 420.1985 & 1 & $\mathrm{C}_{18} \mathrm{H}_{33} \mathrm{~N}_{3} \mathrm{O}_{4} \mathrm{~S}_{2}\left[\mathrm{H}^{+}\right]_{1}$ & -0.06 & $x_{3}$ \\
\hline 519.267 & 1 & $\mathrm{C}_{23} \mathrm{H}_{42} \mathrm{~N}_{4} \mathrm{O}_{5} \mathrm{~S}_{2}\left[\mathrm{H}^{+}\right]_{1}$ & 0.04 & $x_{4}$ \\
\hline 618.3356 & 1 & $\mathrm{C}_{28} \mathrm{H}_{51} \mathrm{~N}_{5} \mathrm{O}_{6} \mathrm{~S}_{2}\left[\mathrm{H}^{+}\right]_{1}$ & 0.43 & $x_{5}$ \\
\hline 717.4034 & 1 & $\mathrm{C}_{33} \mathrm{H}_{60} \mathrm{~N}_{6} \mathrm{O}_{7} \mathrm{~S}_{2}\left[\mathrm{H}^{+}\right]_{1}$ & -0.55 & $x_{6}$ \\
\hline 816.471 & 1 & $\mathrm{C}_{38} \mathrm{H}_{69} \mathrm{~N}_{7} \mathrm{O}_{8} \mathrm{~S}_{2}\left[\mathrm{H}^{+}\right]_{1}$ & -1.50 & $x_{7}$ \\
\hline 915.5407 & 1 & $\mathrm{C}_{43} \mathrm{H}_{78} \mathrm{~N}_{8} \mathrm{O}_{9} \mathrm{~S}_{2}\left[\mathrm{H}^{+}\right]_{1}$ & 0.06 & $x_{8}$ \\
\hline 1014.609 & 1 & $\mathrm{C}_{48} \mathrm{H}_{87} \mathrm{~N}_{9} \mathrm{O}_{10} \mathrm{~S}_{2}\left[\mathrm{H}^{+}\right]_{1}$ & 0.04 & $x_{9}$ \\
\hline 1113.678 & 1 & $\mathrm{C}_{53} \mathrm{H}_{96} \mathrm{~N}_{10} \mathrm{O}_{11} \mathrm{~S}_{2}\left[\mathrm{H}^{+}\right]_{1}$ & 0.18 & $x_{10}$ \\
\hline 1212.746 & 1 & $\mathrm{C}_{58} \mathrm{H}_{105} \mathrm{~N}_{11} \mathrm{O}_{12} \mathrm{~S}_{2}\left[\mathrm{H}^{+}\right]_{1}$ & -0.16 & $x_{11}$ \\
\hline 1311.814 & 1 & $\mathrm{C}_{63} \mathrm{H}_{114} \mathrm{~N}_{12} \mathrm{O}_{13} \mathrm{~S}_{2}\left[\mathrm{H}^{+}\right]_{1}$ & -0.10 & $x_{12}$ \\
\hline 1410.883 & 1 & $\mathrm{C}_{68} \mathrm{H}_{123} \mathrm{~N}_{13} \mathrm{O}_{14} \mathrm{~S}_{2}\left[\mathrm{H}^{+}\right]_{1}$ & 0.34 & $x_{13}$ \\
\hline 1509.95 & 1 & $\mathrm{C}_{73} \mathrm{H}_{132} \mathrm{~N}_{14} \mathrm{O}_{15} \mathrm{~S}_{2}\left[\mathrm{H}^{+}\right]_{1}$ & -0.44 & $x_{14}$ \\
\hline 1609.015 & 1 & $\mathrm{C}_{78} \mathrm{H}_{141} \mathrm{~N}_{15} \mathrm{O}_{16} \mathrm{~S}_{2}\left[\mathrm{H}^{+}\right]_{1}$ & -2.69 & $x_{15}$ \\
\hline 1708.089 & 1 & $\mathrm{C}_{83} \mathrm{H}_{150} \mathrm{~N}_{16} \mathrm{O}_{17} \mathrm{~S}_{2}\left[\mathrm{H}^{+}\right]_{1}$ & 0.51 & $x_{16}$ \\
\hline 1807.158 & 1 & $\mathrm{C}_{88} \mathrm{H}_{159} \mathrm{~N}_{17} \mathrm{O}_{18} \mathrm{~S}_{2}\left[\mathrm{H}^{+}\right]_{1}$ & 1.20 & $x_{17}$ \\
\hline 1906.221 & 1 & $\mathrm{C}_{93} \mathrm{H}_{168} \mathrm{~N}_{18} \mathrm{O}_{19} \mathrm{~S}_{2}\left[\mathrm{H}^{+}\right]_{1}$ & -2.19 & $x_{18}$ \\
\hline \multirow[t]{3}{*}{2005.288} & 1 & $\mathrm{C}_{98} \mathrm{H}_{177} \mathrm{~N}_{19} \mathrm{O}_{20} \mathrm{~S}_{2}\left[\mathrm{H}^{+}\right]_{1}$ & -2.61 & $x_{19}$ \\
\hline & & Absolute average & 0.51 & \\
\hline & & Std Deviation & 0.84 & \\
\hline
\end{tabular}


Supporting Information to Characterisation across a dispersity: polymer mass spectrometry in the second dimension

Table S 3: Mass spectrometry assignments of the 21-mer polyoxazoline end group losses, Figure $1 \mathrm{C}$ in main text

\begin{tabular}{|c|c|c|c|c|}
\hline $\mathrm{m} / \mathrm{z}$ & $q$ & chemical formula & Error & Loss \\
\hline 739.819 & 3 & $\mathrm{C}_{109} \mathrm{H}_{197} \mathrm{~N}_{21} \mathrm{O}_{22} \mathrm{~S}_{2}\left[\mathrm{H}^{+}\right]_{3}$ & -1.39 & Precursor \\
\hline 1109.225 & 2 & $\mathrm{C}_{109} \mathrm{H}_{196} \mathrm{~N}_{21} \mathrm{O}_{22} \mathrm{~S}_{2}\left[\mathrm{H}^{+}\right]_{3}$ & -1.35 & $\mathrm{H}^{*}$ \\
\hline 1095.211 & 2 & $\mathrm{C}_{107} \mathrm{H}_{192} \mathrm{~N}_{21} \mathrm{O}_{22} \mathrm{~S}_{2}\left[\mathrm{H}^{+}\right]_{3}$ & 0.81 & $\mathrm{C}_{2} \mathrm{H}_{5} *$ \\
\hline 1081.214 & 2 & $\mathrm{C}_{106} \mathrm{H}_{192} \mathrm{~N}_{21} \mathrm{O}_{21} \mathrm{~S}_{2}\left[\mathrm{H}^{+}\right]_{3}$ & 1.07 & $\mathrm{C}_{3} \mathrm{H}_{5} \mathrm{O}$ \\
\hline 1080.207 & 2 & $\mathrm{C}_{106} \mathrm{H}_{190} \mathrm{~N}_{21} \mathrm{O}_{21} \mathrm{~S}_{2}\left[\mathrm{H}^{+}\right]_{3}$ & 1.25 & $\mathrm{C}_{3} \mathrm{H}_{7} \mathrm{O}$ \\
\hline 1072.208 & 2 & $\mathrm{C}_{106} \mathrm{H}_{190} \mathrm{~N}_{21} \mathrm{O}_{20} \mathrm{~S}_{2}\left[\mathrm{H}^{+}\right]_{3}$ & 0.13 & $\mathrm{C}_{3} \mathrm{H}_{5} \mathrm{O}-\mathrm{H}_{2} \mathrm{O}$ \\
\hline 1052.685 & 2 & $\mathrm{C}_{103} \mathrm{H}_{185} \mathrm{~N}_{20} \mathrm{O}_{21} \mathrm{~S}_{2}\left[\mathrm{H}^{+}\right]_{3}$ & 1.00 & $\mathrm{C}_{6} \mathrm{H}_{12} \mathrm{NO}$ \\
\hline 1044.198 & 2 & $\mathrm{C}_{103} \mathrm{H}_{186} \mathrm{~N}_{21} \mathrm{O}_{19} \mathrm{~S}_{2}\left[\mathrm{H}^{+}\right]_{3}$ & 2.77 & $\mathrm{C}_{3} \mathrm{H}_{5} \mathrm{O}-\mathrm{C}_{3} \mathrm{H}_{5} \mathrm{O}-\mathrm{OH}$ \\
\hline 1035.226 & 2 & $\mathrm{C}_{104} \mathrm{H}_{188} \mathrm{~N}_{21} \mathrm{O}_{21} \mathrm{~S}_{0}\left[\mathrm{H}^{+}\right]_{3}$ & 0.98 & $\mathrm{C}_{5} \mathrm{H}_{9} \mathrm{OS}_{2}$ \\
\hline 1031.174 & 2 & $\mathrm{C}_{101} \mathrm{H}_{182} \mathrm{~N}_{20} \mathrm{O}_{20} \mathrm{~S}_{2}\left[\mathrm{H}^{+}\right]_{3}$ & -0.71 & $\left(\mathrm{C}_{3} \mathrm{H}_{7} \mathrm{O}\right)\left(\mathrm{C}_{5} \mathrm{H}_{9} \mathrm{NO}\right)$ \\
\hline 985.6924 & 2 & $\mathrm{C}_{99} \mathrm{H}_{179} \mathrm{~N}_{20} \mathrm{O}_{20} \mathrm{~S}_{0}\left[\mathrm{H}^{+}\right]_{3}$ & 1.28 & $\left(\mathrm{C}_{5} \mathrm{H}_{9} \mathrm{OS}_{2}\right)\left(\mathrm{C}_{5} \mathrm{H}_{9} \mathrm{NO}\right)$ \\
\hline 981.6412 & 2 & $\mathrm{C}_{96} \mathrm{H}_{173} \mathrm{~N}_{19} \mathrm{O}_{19} \mathrm{~S}_{2}\left[\mathrm{H}^{+}\right]_{3}$ & 0.42 & $\left(\mathrm{C}_{3} \mathrm{H}_{7} \mathrm{O}\right)_{2}\left(\mathrm{C}_{5} \mathrm{H}_{9} \mathrm{NO}\right)$ \\
\hline 665.7776 & 3 & $\mathrm{C}_{100} \mathrm{H}_{179} \mathrm{~N}_{21} \mathrm{O}_{16} \mathrm{~S}_{2}\left[\mathrm{H}^{+}\right]_{3}$ & -8.61 & ${ }_{3}\left(\mathrm{C}_{3} \mathrm{H}_{5} \mathrm{O}-\mathrm{OH}\right)$ \\
\hline 690.4566 & 3 & $\mathrm{C}_{103} \mathrm{H}_{185} \mathrm{~N}_{21} \mathrm{O}_{18} \mathrm{~S}_{2}\left[\mathrm{H}^{+}\right]_{3}$ & -8.10 & ${ }_{2}\left(\mathrm{C}_{3} \mathrm{H}_{5} \mathrm{O}-\mathrm{OH}\right)$ \\
\hline \multirow[t]{3}{*}{715.1339} & 3 & $\mathrm{C}_{106} \mathrm{H}_{191} \mathrm{~N}_{21} \mathrm{O}_{20} \mathrm{~S}_{2}\left[\mathrm{H}^{+}\right]_{3}$ & -10.10 & $\left(\mathrm{C}_{3} \mathrm{H}_{5} \mathrm{O}-\mathrm{OH}\right)$ \\
\hline & & Absolute Average & 1.08 & \\
\hline & & Standard Deviation & 3.93 & \\
\hline
\end{tabular}


Supporting Information to Characterisation across a dispersity: polymer mass spectrometry in the second dimension

Table S 4: Mass spectrometry assignments of the 24-mer polyoxazoline polymer fragment line, Figure $1 \mathrm{E}$ in main text

\begin{tabular}{|c|c|c|c|c|}
\hline $\mathrm{m} / \mathrm{z}$ & charge & chemical formula & $\begin{array}{l}\text { error } \\
\text { (ppm) }\end{array}$ & assignment \\
\hline 286.2125 & 1 & $\mathrm{C}_{14} \mathrm{H}_{27} \mathrm{~N}_{3} \mathrm{O}_{3} \mathrm{~S}_{0}\left[\mathrm{H}^{+}\right]_{1}$ & 0.04 & $a_{2}$ \\
\hline 385.2803 & 1 & $\mathrm{C}_{19} \mathrm{H}_{36} \mathrm{~N}_{4} \mathrm{O}_{4} \mathrm{~S}_{0}\left[\mathrm{H}^{+}\right]_{1}$ & -1.56 & $a_{3}$ \\
\hline 484.3488 & 1 & $\mathrm{C}_{24} \mathrm{H}_{45} \mathrm{~N}_{5} \mathrm{O}_{5} \mathrm{~S}_{0}\left[\mathrm{H}^{+}\right]_{1}$ & -1.07 & $a_{4}$ \\
\hline 583.4172 & 1 & $\mathrm{C}_{29} \mathrm{H}_{54} \mathrm{~N}_{6} \mathrm{O}_{6} \mathrm{~S}_{0}\left[\mathrm{H}^{+}\right]_{1}$ & -0.91 & $a_{5}$ \\
\hline 682.4857 & 1 & $\mathrm{C}_{34} \mathrm{H}_{63} \mathrm{~N}_{7} \mathrm{O}_{7} \mathrm{~S}_{0}\left[\mathrm{H}^{+}\right]_{1}$ & -0.69 & $a_{6}$ \\
\hline 781.5536 & 1 & $\mathrm{C}_{39} \mathrm{H}_{72} \mathrm{~N}_{8} \mathrm{O}_{8} \mathrm{~S}_{0}\left[\mathrm{H}^{+}\right]_{1}$ & -1.24 & $a_{7}$ \\
\hline 880.6218 & 1 & $\mathrm{C}_{44} \mathrm{H}_{81} \mathrm{~N}_{9} \mathrm{O}_{9} \mathrm{~S}_{0}\left[\mathrm{H}^{+}\right]_{1}$ & -1.32 & $a_{8}$ \\
\hline 979.6896 & 1 & $\mathrm{C}_{49} \mathrm{H}_{90} \mathrm{~N}_{10} \mathrm{O}_{10} \mathrm{~S}_{0}\left[\mathrm{H}^{+}\right]_{1}$ & -1.90 & $a_{9}$ \\
\hline 1078.759 & 1 & $\mathrm{C}_{54} \mathrm{H}_{99} \mathrm{~N}_{11} \mathrm{O}_{11} \mathrm{~S}_{0}\left[\mathrm{H}^{+}\right]_{1}$ & -0.34 & $a_{10}$ \\
\hline 1177.834 & 1 & $\mathrm{C}_{59} \mathrm{H}_{108} \mathrm{~N}_{12} \mathrm{O}_{12} \mathrm{~S}_{0}\left[\mathrm{H}^{+}\right]_{1}$ & 4.56 & $a_{11}$ \\
\hline 1276.897 & 1 & $\mathrm{C}_{64} \mathrm{H}_{117} \mathrm{~N}_{13} \mathrm{O}_{13} \mathrm{~S}_{0}\left[\mathrm{H}^{+}\right]_{1}$ & 0.64 & $a_{12}$ \\
\hline 1375.966 & 1 & $\mathrm{C}_{69} \mathrm{H}_{126} \mathrm{~N}_{14} \mathrm{O}_{14} \mathrm{~S}_{0}\left[\mathrm{H}^{+}\right]_{1}$ & 0.97 & $a_{13}$ \\
\hline 1475.038 & 1 & $\mathrm{C}_{74} \mathrm{H}_{135} \mathrm{~N}_{15} \mathrm{O}_{15} \mathrm{~S}_{0}\left[\mathrm{H}^{+}\right]_{1}$ & 3.22 & $a_{14}$ \\
\hline 1574.102 & 1 & $\mathrm{C}_{79} \mathrm{H}_{144} \mathrm{~N}_{16} \mathrm{O}_{16} \mathrm{~S}_{0}\left[\mathrm{H}^{+}\right]_{1}$ & 0.32 & $a_{15}$ \\
\hline 1673.167 & 1 & $\mathrm{C}_{84} \mathrm{H}_{153} \mathrm{~N}_{17} \mathrm{O}_{17} \mathrm{~S}_{0}\left[\mathrm{H}^{+}\right]_{1}$ & -2.05 & $a_{16}$ \\
\hline 1772.239 & 1 & $\mathrm{C}_{89} \mathrm{H}_{162} \mathrm{~N}_{18} \mathrm{O}_{18} \mathrm{~S}_{0}\left[\mathrm{H}^{+}\right]_{1}$ & 0.11 & $a_{17}$ \\
\hline 1871.3 & 1 & $\mathrm{C}_{94} \mathrm{H}_{171} \mathrm{~N}_{19} \mathrm{O}_{19} \mathrm{~S}_{0}\left[\mathrm{H}^{+}\right]_{1}$ & -3.61 & $a_{18}$ \\
\hline 1970.376 & 1 & $\mathrm{C}_{99} \mathrm{H}_{180} \mathrm{~N}_{20} \mathrm{O}_{20} \mathrm{~S}_{0}\left[\mathrm{H}^{+}\right]_{1}$ & 0.01 & $a_{19}$ \\
\hline 2069.444 & 1 & $\mathrm{C}_{104} \mathrm{H}_{189} \mathrm{~N}_{21} \mathrm{O}_{21} \mathrm{~S}_{0}\left[\mathrm{H}^{+}\right]_{1}$ & 0.23 & $a_{20}$ \\
\hline 2168.51 & 1 & $\mathrm{C}_{109} \mathrm{H}_{198} \mathrm{~N}_{22} \mathrm{O}_{22} \mathrm{~S}_{0}\left[\mathrm{H}^{+}\right]_{1}$ & -1.12 & $a_{21}$ \\
\hline 2267.576 & 1 & $\mathrm{C}_{114} \mathrm{H}_{207} \mathrm{~N}_{23} \mathrm{O}_{23} \mathrm{~S}_{0}\left[\mathrm{H}^{+}\right]_{1}$ & -2.33 & $a_{22}$ \\
\hline 420.1987 & 1 & $\mathrm{C}_{18} \mathrm{H}_{33} \mathrm{~N}_{3} \mathrm{O}_{4} \mathrm{~S}_{2}\left[\mathrm{H}^{+}\right]_{1}$ & 0.32 & $x_{3}$ \\
\hline 519.2661 & 1 & $\mathrm{C}_{23} \mathrm{H}_{42} \mathrm{~N}_{4} \mathrm{O}_{5} \mathrm{~S}_{2}\left[\mathrm{H}^{+}\right]_{1}$ & -1.54 & $x_{4}$ \\
\hline 618.3362 & 1 & $\mathrm{C}_{28} \mathrm{H}_{51} \mathrm{~N}_{5} \mathrm{O}_{6} \mathrm{~S}_{2}\left[\mathrm{H}^{+}\right]_{1}$ & 1.31 & $x_{5}$ \\
\hline 717.4038 & 1 & $\mathrm{C}_{33} \mathrm{H}_{60} \mathrm{~N}_{6} \mathrm{O}_{7} \mathrm{~S}_{2}\left[\mathrm{H}^{+}\right]_{1}$ & 0.05 & $x_{6}$ \\
\hline 816.4742 & 1 & $\mathrm{C}_{38} \mathrm{H}_{69} \mathrm{~N}_{7} \mathrm{O}_{8} \mathrm{~S}_{2}\left[\mathrm{H}^{+}\right]_{1}$ & 2.41 & $x_{7}$ \\
\hline 915.5418 & 1 & $\mathrm{C}_{43} \mathrm{H}_{78} \mathrm{~N}_{8} \mathrm{O}_{9} \mathrm{~S}_{2}\left[\mathrm{H}^{+}\right]_{1}$ & 1.34 & $x_{8}$ \\
\hline 1014.608 & 1 & $\mathrm{C}_{48} \mathrm{H}_{87} \mathrm{~N}_{9} \mathrm{O}_{10} \mathrm{~S}_{2}\left[\mathrm{H}^{+}\right]_{1}$ & -0.83 & $x_{9}$ \\
\hline 1113.674 & 1 & $\mathrm{C}_{53} \mathrm{H}_{96} \mathrm{~N}_{10} \mathrm{O}_{11} \mathrm{~S}_{2}\left[\mathrm{H}^{+}\right]_{1}$ & -2.80 & $x_{10}$ \\
\hline 1212.744 & 1 & $\mathrm{C}_{58} \mathrm{H}_{105} \mathrm{~N}_{11} \mathrm{O}_{12} \mathrm{~S}_{2}\left[\mathrm{H}^{+}\right]_{1}$ & -1.46 & $x_{11}$ \\
\hline 1311.804 & 1 & $\mathrm{C}_{63} \mathrm{H}_{114} \mathrm{~N}_{12} \mathrm{O}_{13} \mathrm{~S}_{2}\left[\mathrm{H}^{+}\right]_{1}$ & -7.52 & $x_{12}$ \\
\hline 1410.885 & 1 & $\mathrm{C}_{68} \mathrm{H}_{123} \mathrm{~N}_{13} \mathrm{O}_{14} \mathrm{~S}_{2}\left[\mathrm{H}^{+}\right]_{1}$ & 1.32 & $x_{13}$ \\
\hline 1609.025 & 1 & $\mathrm{C}_{78} \mathrm{H}_{141} \mathrm{~N}_{15} \mathrm{O}_{16} \mathrm{~S}_{2}\left[\mathrm{H}^{+}\right]_{1}$ & 3.48 & $x_{15}$ \\
\hline 1708.077 & 1 & $\mathrm{C}_{83} \mathrm{H}_{150} \mathrm{~N}_{16} \mathrm{O}_{17} \mathrm{~S}_{2}\left[\mathrm{H}^{+}\right]_{1}$ & -6.49 & $x_{16}$ \\
\hline 1807.153 & 1 & $\mathrm{C}_{88} \mathrm{H}_{159} \mathrm{~N}_{17} \mathrm{O}_{18} \mathrm{~S}_{2}\left[\mathrm{H}^{+}\right]_{1}$ & -1.69 & $x_{17}$ \\
\hline 1906.226 & 1 & $\mathrm{C}_{93} \mathrm{H}_{168} \mathrm{~N}_{18} \mathrm{O}_{19} \mathrm{~S}_{2}\left[\mathrm{H}^{+}\right]_{1}$ & 0.67 & $x_{18}$ \\
\hline 2104.363 & 1 & $\mathrm{C}_{103} \mathrm{H}_{186} \mathrm{~N}_{20} \mathrm{O}_{21} \mathrm{~S}_{2}\left[\mathrm{H}^{+}\right]_{1}$ & 0.53 & $x_{20}$ \\
\hline 2203.421 & 1 & $\mathrm{C}_{108} \mathrm{H}_{195} \mathrm{~N}_{21} \mathrm{O}_{22} \mathrm{~S}_{2}\left[\mathrm{H}^{+}\right]_{1}$ & -3.92 & $x_{21}$ \\
\hline \multirow[t]{3}{*}{2302.503} & 1 & $\mathrm{C}_{113} \mathrm{H}_{204} \mathrm{~N}_{22} \mathrm{O}_{23} \mathrm{~S}_{2}\left[\mathrm{H}^{+}\right]_{1}$ & 2.20 & $x_{22}$ \\
\hline & & Absolute Average & 1.75 & \\
\hline & & Standard Deviation & 2.30 & \\
\hline
\end{tabular}


Supporting Information to Characterisation across a dispersity: polymer mass spectrometry in the second dimension

Table S 5: Mass spectrometry assignments of the 24-mer polyoxazoline end group losses, Figure 1E in main text

\begin{tabular}{|c|c|c|c|c|}
\hline$m / z$ & q & Chemical & oss & error (ppm) \\
\hline 838.88604 & 3 & $\mathrm{C}_{124} \mathrm{H}_{224} \mathrm{~N}_{24} \mathrm{O}_{25} \mathrm{~S}_{2}\left[\mathrm{H}^{+}\right]_{3}$ & Precursor & -2.90 \\
\hline 1192.79635 & 2 & $\mathrm{C}_{118} \mathrm{H}_{214} \mathrm{~N}_{24} \mathrm{O}_{22} \mathrm{~S}_{2}\left[\mathrm{H}^{+}\right]_{2}$ & $\mathrm{C}_{3} \mathrm{H}_{5} \mathrm{O}-\mathrm{C}_{3} \mathrm{H}_{5} \mathrm{O}-\mathrm{OH}$ & -1.03 \\
\hline 1134.29332 & 2 & $\mathrm{C}_{114} \mathrm{H}_{206} \mathrm{~N}_{23} \mathrm{O}_{23} \mathrm{~S}_{0}\left[\mathrm{H}^{+}\right]_{3}$ & $\left(\mathrm{C}_{5} \mathrm{H}_{9} \mathrm{OS}_{2}\right)\left(\mathrm{C}_{5} \mathrm{H}_{9} \mathrm{NO}\right)$ & -0.39 \\
\hline 1243.81274 & 2 & $\mathrm{C}_{122} \mathrm{H}_{219} \mathrm{~N}_{24} \mathrm{O}_{25} \mathrm{~S}_{2}\left[\mathrm{H}^{+}\right]_{3}$ & $\mathrm{C}_{2} \mathrm{H}_{5}^{*}$ & -0.33 \\
\hline 1151.7522 & 2 & $\mathrm{C}_{113} \mathrm{H}_{203} \mathrm{~N}_{22} \mathrm{O}_{23} \mathrm{~S}_{2}\left[\mathrm{H}^{+}\right]_{3}$ & $\left(\mathrm{C}_{5} \mathrm{H}_{9} \mathrm{NO}\right)\left(\mathrm{C}_{6} \mathrm{H}_{12} \mathrm{NO}\right)$ & -0.31 \\
\hline 1201.28664 & 2 & $\mathrm{C}_{118} \mathrm{H}_{212} \mathrm{~N}_{23} \mathrm{O}_{24} \mathrm{~S}_{2}\left[\mathrm{H}^{+}\right]_{3}$ & $\mathrm{C}_{6} \mathrm{H}_{12} \mathrm{NO}$ & -0.10 \\
\hline 1249.32743 & 2 & $\mathrm{C}_{124} \mathrm{H}_{222} \mathrm{~N}_{24} \mathrm{O}_{24} \mathrm{~S}_{2}\left[\mathrm{H}^{+}\right]_{3}$ & $\mathrm{H}_{2} \mathrm{O}$ & 0.00 \\
\hline 1228.80789 & 2 & $\mathrm{C}_{121} \mathrm{H}_{217} \mathrm{~N}_{24} \mathrm{O}_{24} \mathrm{~S}_{2}\left[\mathrm{H}^{+}\right]_{3}$ & $\mathrm{C}_{3} \mathrm{H}_{7} \mathrm{O}$ & 0.02 \\
\hline 1213.82972 & 2 & $\mathrm{C}_{121} \mathrm{H}_{219} \mathrm{~N}_{24} \mathrm{O}_{24} \mathrm{~S}_{1}\left[\mathrm{H}^{+}\right]_{3}$ & $\mathrm{C}_{3} \mathrm{H}_{5} \mathrm{OS}$ & 0.05 \\
\hline 1229.81612 & 2 & $\mathrm{C}_{121} \mathrm{H}_{219} \mathrm{~N}_{24} \mathrm{O}_{24} \mathrm{~S}_{2}\left[\mathrm{H}^{+}\right]_{3}$ & $\mathrm{C}_{3} \mathrm{H}_{5} \mathrm{O}$ & 0.35 \\
\hline 1084.76043 & 2 & $\mathrm{C}_{109} \mathrm{H}_{198} \mathrm{~N}_{22} \mathrm{O}_{22} \mathrm{~S}_{0}\left[\mathrm{H}^{+}\right]_{2}$ & $\left(\mathrm{C}_{5} \mathrm{H}_{9} \mathrm{OS}_{2}\right) 2\left(\mathrm{C}_{5} \mathrm{H}_{9} \mathrm{NO}\right)$ & 0.55 \\
\hline 1179.27431 & 2 & $\mathrm{C}_{116} \mathrm{H}_{208} \mathrm{~N}_{23} \mathrm{O}_{23} \mathrm{~S}_{2}\left[\mathrm{H}^{+}\right]_{3}$ & $\left(\mathrm{C}_{3} \mathrm{H}_{7} \mathrm{O}\right)\left(\mathrm{C}_{5} \mathrm{H}_{9} \mathrm{NO}\right)$ & 0.55 \\
\hline 1102.21904 & 2 & $\mathrm{C}_{108} \mathrm{H}_{194} \mathrm{~N}_{21} \mathrm{O}_{22} \mathrm{~S}_{2}\left[\mathrm{H}^{+}\right]_{3}$ & $\left(\mathrm{C}_{5} \mathrm{H}_{9} \mathrm{NO}\right) 2\left(\mathrm{C}_{6} \mathrm{H}_{12} \mathrm{NO}\right)$ & 0.62 \\
\hline 1220.81197 & 2 & $\mathrm{C}_{121} \mathrm{H}_{217} \mathrm{~N}_{24} \mathrm{O}_{23} \mathrm{~S}_{2}\left[\mathrm{H}^{+}\right]_{3}$ & $\mathrm{C}_{3} \mathrm{H}_{5} \mathrm{O}-\mathrm{H}_{2} \mathrm{O}$ & 1.28 \\
\hline 1183.8304 & 2 & $\mathrm{C}_{119} \mathrm{H}_{216} \mathrm{~N}_{24} \mathrm{O}_{24} \mathrm{~S}_{0}\left[\mathrm{H}^{+}\right]_{2}$ & $\mathrm{C}_{5} \mathrm{H}_{9} \mathrm{OS}_{2}$ & 1.82 \\
\hline 764.85017 & 3 & $\mathrm{C}_{115} \mathrm{H}_{206} \mathrm{~N}_{24} \mathrm{O}_{19} \mathrm{~S}_{2}\left[\mathrm{H}^{+}\right]_{3}$ & $3\left(\mathrm{C}_{3} \mathrm{H}_{5} \mathrm{O}-\mathrm{OH}\right)$ & -1.99 \\
\hline 789.5289 & 3 & $\mathrm{C}_{118} \mathrm{H}_{212} \mathrm{~N}_{24} \mathrm{O}_{21} \mathrm{~S}_{2}\left[\mathrm{H}^{+}\right]_{3}$ & $2\left(\mathrm{C}_{3} \mathrm{H}_{5} \mathrm{O}-\mathrm{OH}\right)$ & -2.18 \\
\hline \multirow[t]{3}{*}{814.20795} & 3 & $\mathrm{C}_{121} \mathrm{H}_{218} \mathrm{~N}_{24} \mathrm{O}_{23} \mathrm{~S}_{2}\left[\mathrm{H}^{+}\right]_{3}$ & $\left(\mathrm{C}_{3} \mathrm{H}_{5} \mathrm{O}-\mathrm{OH}\right)$ & -1.96 \\
\hline & & & Absolute Average & 0.42 \\
\hline & & & Standard Deviation & 1.21 \\
\hline
\end{tabular}


Supporting Information to Characterisation across a dispersity: polymer mass spectrometry in the second dimension

Table S 6: Mass spectrometry assignments of the H-terminated Polyoxazoline fragments Figure 2 in Main text

$\mathrm{m} / \mathbf{z}$
1193.28683
1187.27058
1173.27314
1157.28703
1127.79022
1102.72245
1077.752

charge

loss

$2 \mathrm{H}^{\bullet}$

error (ppm)

$2 \mathrm{CH}_{3} \cdot$

$-2.08$

$2 \mathrm{H}_{2} \mathrm{O}$

$-0.45$

$2 \mathrm{C}_{3} \mathrm{H}_{5} \mathrm{O}^{\cdot}$

$-0.44$

$2 \mathrm{C}_{5} \mathrm{H}_{9} \mathrm{OS}_{2}$

$-0.51$

$2 \mathrm{C}_{10} \mathrm{H}_{18} \mathrm{~N}_{2} \mathrm{O}_{2}$

0.33

$2 \mathrm{C}_{10} \mathrm{H}_{18} \mathrm{NO}_{2} \mathrm{~S}_{2}$

0.17

Absolute Average

0.25

Standard Deviation

0.11

0.77

\begin{tabular}{crlrr}
$\mathbf{m} / \mathbf{z}$ & charge & \multicolumn{1}{c}{ Formula } & error (ppm) & Assignment \\
1361.9492 & 1 & $\mathrm{C}_{68} \mathrm{H}_{124} \mathrm{~N}_{14} \mathrm{O}_{14}\left[\mathrm{H}^{+}\right]_{1}$ & -0.16 & $a_{14}$ \\
1461.01685 & 1 & $\mathrm{C}_{73} \mathrm{H}_{133} \mathrm{~N}_{15} \mathrm{O}_{15}\left[\mathrm{H}^{+}\right]_{1}$ & -0.67 & $a_{15}$ \\
1560.08682 & 1 & $\mathrm{C}_{78} \mathrm{H}_{142} \mathrm{~N}_{16} \mathrm{O}_{16}\left[\mathrm{H}^{+}\right]_{1}$ & 0.37 & $a_{16}$ \\
1659.15588 & 1 & $\mathrm{C}_{83} \mathrm{H}_{151} \mathrm{~N}_{17} \mathrm{O}_{17}\left[\mathrm{H}^{+}\right]_{1}$ & 0.73 & $a_{17}$ \\
1758.21824 & 1 & $\mathrm{C}_{88} \mathrm{H}_{160} \mathrm{~N}_{18} \mathrm{O}_{18}\left[\mathrm{H}^{+}\right]_{1}$ & -2.75 & $a_{18}$ \\
1857.28664 & 1 & $\mathrm{C}_{93} \mathrm{H}_{169} \mathrm{~N}_{19} \mathrm{O}_{19}\left[\mathrm{H}^{+}\right]_{1}$ & -2.61 & $a_{19}$ \\
1956.35654 & 1 & $\mathrm{C}_{98} \mathrm{H}_{178} \mathrm{~N}_{20} \mathrm{O}_{20}\left[\mathrm{H}^{+}\right]_{1}$ & -1.72 & $a_{20}$ \\
2055.42795 & 1 & $\mathrm{C}_{103} \mathrm{H}_{187} \mathrm{~N}_{21} \mathrm{O}_{21}\left[\mathrm{H}^{+}\right]_{1}$ & -0.18 & $a_{21}$ \\
& & Absolute Average & 0.23 &
\end{tabular}


Supporting Information to Characterisation across a dispersity: polymer mass spectrometry in the second dimension

Table S 7: Mass spectrometry assignments of the polyacrylamide fragments Figure 5 in Main text

\begin{tabular}{|c|c|c|c|c|}
\hline $\mathrm{m} / \mathrm{z}$ & charge & chemical formula & error (ppm) & assignment \\
\hline 186.13603 & 1 & $\mathrm{C}_{9} \mathrm{H}_{17} \mathrm{~N}_{2} \mathrm{O}_{2}\left[\mathrm{H}^{+}\right]_{1}$ & -1.34 & $j_{2}$ \\
\hline 285.20459 & 1 & $\mathrm{C}_{14} \mathrm{H}_{26} \mathrm{~N}_{3} \mathrm{O}_{3}\left[\mathrm{H}^{+}\right]_{1}$ & -0.36 & $j_{3}$ \\
\hline 384.2731 & 1 & $\mathrm{C}_{19} \mathrm{H}_{35} \mathrm{~N}_{4} \mathrm{O}_{4}\left[\mathrm{H}^{+}\right]_{1}$ & -0.02 & $j_{4}$ \\
\hline 483.34156 & 1 & $\mathrm{C}_{24} \mathrm{H}_{44} \mathrm{~N}_{5} \mathrm{O}_{5}\left[\mathrm{H}^{+}\right]_{1}$ & 0.08 & $j_{5}$ \\
\hline 582.41007 & 1 & $\mathrm{C}_{29} \mathrm{H}_{53} \mathrm{~N}_{6} \mathrm{O}_{6}\left[\mathrm{H}^{+}\right]_{1}$ & 0.23 & $j_{6}$ \\
\hline 681.47816 & 1 & $\mathrm{C}_{34} \mathrm{H}_{62} \mathrm{~N}_{7} \mathrm{O}_{7}\left[\mathrm{H}^{+}\right]_{1}$ & -0.28 & $j_{7}$ \\
\hline 780.54674 & 1 & $\mathrm{C}_{39} \mathrm{H}_{71} \mathrm{~N}_{8} \mathrm{O}_{8}\left[\mathrm{H}^{+}\right]_{1}$ & -0.03 & $j_{8}$ \\
\hline 879.61432 & 1 & $\mathrm{C}_{44} \mathrm{H}_{80} \mathrm{~N}_{9} \mathrm{O}_{9}\left[\mathrm{H}^{+}\right]_{1}$ & -0.97 & $j_{g}$ \\
\hline 978.68843 & 1 & $\mathrm{C}_{49} \mathrm{H}_{89} \mathrm{~N}_{10} \mathrm{O}_{10}\left[\mathrm{H}^{+}\right]_{1}$ & 4.94 & $j_{10}$ \\
\hline 199.1441 & 1 & $\mathrm{C}_{10} \mathrm{H}_{18} \mathrm{~N}_{2} \mathrm{O}_{2}\left[\mathrm{H}^{+}\right]_{1}$ & -0.02 & $k_{2}$ \\
\hline 298.21228 & 1 & $\mathrm{C}_{15} \mathrm{H}_{27} \mathrm{~N}_{3} \mathrm{O}_{3}\left[\mathrm{H}^{+}\right]_{1}$ & -0.80 & $k_{3}$ \\
\hline 397.28111 & 1 & $\mathrm{C}_{20} \mathrm{H}_{36} \mathrm{~N}_{4} \mathrm{O}_{4}\left[\mathrm{H}^{+}\right]_{1}$ & 0.45 & $k_{4}$ \\
\hline 496.34935 & 1 & $\mathrm{C}_{25} \mathrm{H}_{45} \mathrm{~N}_{5} \mathrm{O}_{5}\left[\mathrm{H}^{+}\right]_{1}$ & 0.01 & $k_{5}$ \\
\hline 595.418 & 1 & $\mathrm{C}_{30} \mathrm{H}_{54} \mathrm{~N}_{6} \mathrm{O}_{6}\left[\mathrm{H}^{+}\right]_{1}$ & 0.40 & $k_{6}$ \\
\hline 694.48636 & 1 & $\mathrm{C}_{35} \mathrm{H}_{63} \mathrm{~N}_{7} \mathrm{O}_{7}\left[\mathrm{H}^{+}\right]_{1}$ & 0.27 & $k_{7}$ \\
\hline 793.55517 & 1 & $\mathrm{C}_{40} \mathrm{H}_{72} \mathrm{~N}_{8} \mathrm{O}_{8}\left[\mathrm{H}^{+}\right]_{1}$ & 0.73 & $k_{8}$ \\
\hline 892.62482 & 1 & $\mathrm{C}_{45} \mathrm{H}_{81} \mathrm{~N}_{9} \mathrm{O}_{9}\left[\mathrm{H}^{+}\right]_{1}$ & 2.04 & $k_{g}$ \\
\hline 1090.7603 & 1 & $\mathrm{C}_{55} \mathrm{H}_{99} \mathrm{~N}_{11} \mathrm{O}_{11}\left[\mathrm{H}^{+}\right]_{1}$ & 0.43 & $k_{11}$ \\
\hline 1189.82803 & 1 & $\mathrm{C}_{60} \mathrm{H}_{108} \mathrm{~N}_{12} \mathrm{O}_{12}\left[\mathrm{H}^{+}\right]_{1}$ & -0.18 & $k_{12}$ \\
\hline 1288.89665 & 1 & $\mathrm{C}_{65} \mathrm{H}_{117} \mathrm{~N}_{13} \mathrm{O}_{13}\left[\mathrm{H}^{+}\right]_{1}$ & -0.01 & $k_{13}$ \\
\hline 1387.96489 & 1 & $\mathrm{C}_{70} \mathrm{H}_{126} \mathrm{~N}_{14} \mathrm{O}_{14}\left[\mathrm{H}^{+}\right]_{1}$ & -0.13 & $k_{14}$ \\
\hline 1487.03415 & 1 & $\mathrm{C}_{75} \mathrm{H}_{135} \mathrm{~N}_{15} \mathrm{O}_{15}\left[\mathrm{H}^{+}\right]_{1}$ & 0.45 & $k_{15}$ \\
\hline 1586.10609 & 1 & $\mathrm{C}_{80} \mathrm{H}_{144} \mathrm{~N}_{16} \mathrm{O}_{16}\left[\mathrm{H}^{+}\right]_{1}$ & 2.64 & $k_{16}$ \\
\hline 1685.17033 & 1 & $\mathrm{C}_{85} \mathrm{H}_{153} \mathrm{~N}_{17} \mathrm{O}_{17}\left[\mathrm{H}^{+}\right]_{1}$ & 0.01 & $k_{17}$ \\
\hline 285.18069 & 1 & $\mathrm{C}_{14} \mathrm{H}_{24} \mathrm{~N}_{2} \mathrm{O}_{4}\left[\mathrm{H}^{+}\right]_{1}$ & -0.68 & $a_{2}$ \\
\hline 384.24935 & 1 & $\mathrm{C}_{19} \mathrm{H}_{33} \mathrm{~N}_{3} \mathrm{O}_{5}\left[\mathrm{H}^{+}\right]_{1}$ & 0.14 & $a_{3}$ \\
\hline 483.31772 & 1 & $\mathrm{C}_{24} \mathrm{H}_{42} \mathrm{~N}_{4} \mathrm{O}_{6}\left[\mathrm{H}^{+}\right]_{1}$ & 0.02 & $a_{4}$ \\
\hline 582.38707 & 1 & $\mathrm{C}_{29} \mathrm{H}_{51} \mathrm{~N}_{5} \mathrm{O}_{7}\left[\mathrm{H}^{+}\right]_{1}$ & 1.62 & $a_{5}$ \\
\hline 681.45524 & 1 & $\mathrm{C}_{34} \mathrm{H}_{60} \mathrm{~N}_{6} \mathrm{O}_{8}\left[\mathrm{H}^{+}\right]_{1}$ & 1.03 & $a_{6}$ \\
\hline 780.52254 & 1 & $\mathrm{C}_{39} \mathrm{H}_{69} \mathrm{~N}_{7} \mathrm{O}_{9}\left[\mathrm{H}^{+}\right]_{1}$ & -0.53 & $a_{7}$ \\
\hline 879.59037 & 1 & $\mathrm{C}_{44} \mathrm{H}_{78} \mathrm{~N}_{8} \mathrm{O}_{10}\left[\mathrm{H}^{+}\right]_{1}$ & -1.13 & $a_{8}$ \\
\hline 1474.00461 & 1 & $\mathrm{C}_{74} \mathrm{H}_{132} \mathrm{~N}_{14} \mathrm{O}_{16}\left[\mathrm{H}^{+}\right]_{1}$ & 1.87 & $a_{14}$ \\
\hline 1573.07149 & 1 & $\mathrm{C}_{79} \mathrm{H}_{141} \mathrm{~N}_{15} \mathrm{O}_{17}\left[\mathrm{H}^{+}\right]_{1}$ & 0.78 & $a_{15}$ \\
\hline 1672.14274 & 1 & $\mathrm{C}_{84} \mathrm{H}_{150} \mathrm{~N}_{16} \mathrm{O}_{18}\left[\mathrm{H}^{+}\right]_{1}$ & 2.43 & $a_{16}$ \\
\hline 1771.20696 & 1 & $\mathrm{C}_{89} \mathrm{H}_{159} \mathrm{~N}_{17} \mathrm{O}_{19}\left[\mathrm{H}^{+}\right]_{1}$ & -0.07 & $a_{17}$ \\
\hline 272.17301 & 1 & $\mathrm{C}_{13} \mathrm{H}_{23} \mathrm{~N}_{2} \mathrm{O}_{4}\left[\mathrm{H}^{+}\right]_{1}$ & -0.18 & $b_{2}$ \\
\hline 371.24152 & 1 & $\mathrm{C}_{18} \mathrm{H}_{32} \mathrm{~N}_{3} \mathrm{O}_{5}\left[\mathrm{H}^{+}\right]_{1}$ & 0.13 & $b_{3}$ \\
\hline 470.30993 & 1 & $\mathrm{C}_{23} \mathrm{H}_{41} \mathrm{~N}_{4} \mathrm{O}_{6}\left[\mathrm{H}^{+}\right]_{1}$ & 0.09 & $b_{4}$ \\
\hline 569.37845 & 1 & $\mathrm{C}_{28} \mathrm{H}_{50} \mathrm{~N}_{5} \mathrm{O}_{7}\left[\mathrm{H}^{+}\right]_{1}$ & 0.26 & $b_{5}$ \\
\hline 668.44731 & 1 & $\mathrm{C}_{33} \mathrm{H}_{59} \mathrm{~N}_{6} \mathrm{O}_{8}\left[\mathrm{H}^{+}\right]_{1}$ & 0.89 & $b_{6}$ \\
\hline 767.51671 & 1 & $\mathrm{C}_{38} \mathrm{H}_{68} \mathrm{~N}_{7} \mathrm{O}_{9}\left[\mathrm{H}^{+}\right]_{1}$ & 2.06 & $b_{7}$ \\
\hline 866.58274 & 1 & $\mathrm{C}_{43} \mathrm{H}_{77} \mathrm{~N}_{8} \mathrm{O}_{10}\left[\mathrm{H}^{+}\right]_{1}$ & -0.93 & $b_{8}$ \\
\hline 965.64807 & 1 & $\mathrm{C}_{48} \mathrm{H}_{86} \mathrm{~N}_{9} \mathrm{O}_{11}\left[\mathrm{H}^{+}\right]_{1}$ & -4.02 & $b_{9}$ \\
\hline
\end{tabular}


Supporting Information to Characterisation across a dispersity: polymer mass spectrometry in the second dimension

\begin{tabular}{|c|c|c|c|c|c|}
\hline \multicolumn{2}{|l|}{1064.72091} & 1 & $\mathrm{C}_{53} \mathrm{H}_{95} \mathrm{~N}_{10} \mathrm{O}_{12}\left[\mathrm{H}^{+}\right]_{1}$ & 0.51 & $b_{10}$ \\
\hline \multicolumn{2}{|l|}{1163.78504} & 1 & $\mathrm{C}_{58} \mathrm{H}_{104} \mathrm{~N}_{11} \mathrm{O}_{13}\left[\mathrm{H}^{+}\right]_{1}$ & -3.22 & $b_{11}$ \\
\hline \multicolumn{2}{|l|}{1262.85706} & 1 & $\mathrm{C}_{63} \mathrm{H}_{113} \mathrm{~N}_{12} \mathrm{O}_{14}\left[\mathrm{H}^{+}\right]_{1}$ & -0.11 & $b_{12}$ \\
\hline \multicolumn{2}{|l|}{1361.92267} & 1 & $\mathrm{C}_{68} \mathrm{H}_{122} \mathrm{~N}_{13} \mathrm{O}_{15}\left[\mathrm{H}^{+}\right]_{1}$ & -2.16 & $b_{13}$ \\
\hline \multicolumn{2}{|l|}{1460.99457} & 1 & $\mathrm{C}_{73} \mathrm{H}_{131} \mathrm{~N}_{14} \mathrm{O}_{16}\left[\mathrm{H}^{+}\right]_{1}$ & 0.37 & $b_{14}$ \\
\hline \multirow{3}{*}{\multicolumn{2}{|c|}{1560.05948}} & 1 & $\mathrm{C}_{78} \mathrm{H}_{140} \mathrm{~N}_{15} \mathrm{O}_{17}\left[\mathrm{H}^{+}\right]_{1}$ & -1.90 & $b_{15}$ \\
\hline & & & Average & 0.90 & \\
\hline & & & Standard deviation & 1.41 & \\
\hline 999.67541 & 2 & & $\mathrm{C}_{100} \mathrm{H}_{178} \mathrm{~N}_{19} \mathrm{O}_{22}\left[\mathrm{H}^{+}\right]_{2}$ & -1.56 & $-\mathrm{C}_{3} \mathrm{H}_{9} \mathrm{~N}$ \\
\hline 1021.70275 & 2 & & $\mathrm{C}_{102} \mathrm{H}_{184} \mathrm{~N}_{20} \mathrm{O}_{22}\left[\mathrm{H}^{+}\right]_{2}$ & 0.75 & $-\mathrm{CH}_{3}$ \\
\hline 626.06488 & 3 & & $\mathrm{C}_{95} \mathrm{H}_{158} \mathrm{~N}_{16} \mathrm{O}_{22}\left[\mathrm{H}^{+}\right]_{3}$ & -0.45 & Precursor - $4\left(\mathrm{HNC}_{2} \mathrm{H}_{6}\right)$ \\
\hline 641.08408 & 3 & & $\mathrm{C}_{97} \mathrm{H}_{165} \mathrm{~N}_{17} \mathrm{O}_{22}\left[\mathrm{H}^{+}\right]_{3}$ & -0.57 & Precursor - $3\left(\mathrm{HNC}_{2} \mathrm{H}_{6}\right)$ \\
\hline 656.10339 & 3 & & $\mathrm{C}_{99} \mathrm{H}_{172} \mathrm{~N}_{18} \mathrm{O}_{22}\left[\mathrm{H}^{+}\right]_{3}$ & -0.52 & Precursor - $2\left(\mathrm{HNC}_{2} \mathrm{H}_{6}\right)$ \\
\hline 671.1226 & 3 & & $\mathrm{C}_{101} \mathrm{H}_{179} \mathrm{~N}_{19} \mathrm{O}_{22}\left[\mathrm{H}^{+}\right]_{3}$ & -0.61 & Precursor - $\mathrm{HNC}_{2} \mathrm{H}_{6}$ \\
\hline 686.14151 & 3 & & $\mathrm{C}_{103} \mathrm{H}_{186} \mathrm{~N}_{20} \mathrm{O}_{22}\left[\mathrm{H}^{+}\right]_{3}$ & -1.15 & Precursor \\
\hline
\end{tabular}

